\title{
Stochastic Attractors for Shell Phenomenological Models of Turbulence
}

\author{
Hakima Bessaih • Franco Flandoli · Edriss S. Titi
}

Received: 26 October 2009 / Accepted: 14 June 2010 / Published online: 9 July 2010

(C) The Author(s) 2010. This article is published with open access at Springerlink.com

\begin{abstract}
Recently, it has been proposed that the Navier-Stokes equations and a relevant linear advection model have the same long-time statistical properties, in particular, they have the same scaling exponents of their structure functions. This assertion has been investigate rigorously in the context of certain nonlinear deterministic phenomenological shell model, the Sabra shell model, of turbulence and its corresponding linear advection counterpart model. This relationship has been established through a "homotopy-like" coefficient $\lambda$ which bridges continuously between the two systems. That is, for $\lambda=1$ one obtains the full nonlinear model, and the corresponding linear advection model is achieved for $\lambda=0$. In this paper, we investigate the validity of this assertion for certain stochastic phenomenological shell models of turbulence driven by an additive noise. We prove the continuous dependence of the solutions with respect to the parameter $\lambda$. Moreover, we show the existence of a finite-dimensional random attractor for each value of $\lambda$ and establish the upper semicontinuity property of this random attractors, with respect to the parameter $\lambda$. This property is proved by a pathwise argument. Our study aims toward the development of basic results and techniques that may contribute to the understanding of the relation between the long-time statistical properties of the nonlinear and linear models.
\end{abstract}

\footnotetext{
H. Bessaih

Department of Mathematics, University of Wyoming, Dept. 3036, 1000 East University Avenue, Laramie, WY 82071, USA

e-mail: bessaih@uwyo.edu

F. Flandoli ( $\square)$

Dipartimento di Matematica applicata “U. Dini”, Università di Pisa, Via Buonarrotti 1, 56127 Pisa, Italy e-mail: flandoli@dma.unipi.it
}

E.S. Titi

Department of Mathematics and Department of Mechanical and Aerospace Engineering, University of California, Irvine, CA 92697, USA

e-mail: etiti@math.uci.edu

E.S. Titi

Department of Computer Science and Applied Mathematics, The Weizmann Institute of Science,

Rehovot 76100, Israel 
Keywords Shell models of turbulence · Turbulence models · Passive scalar · Linear advection models $\cdot$ Random dynamical systems $\cdot$ Stochastic analysis

\section{Introduction}

\subsection{Motivation}

The GOY shell model [29] and [35], and Sabra shell model [32] are some of the most interesting and most popular examples of simplified phenomenological models of turbulence. This is because, although departing from reality, they capture some essential statistical properties and features of turbulent flows, like the energy and the enstrophy cascade, and the power law decay of the structure functions in some range of wave numbers-the inertial range. We refer the reader to, e.g., [2, 7, 10, 12, 27, 28], and references therein for several descriptions and results. Often, in numerical or theoretical investigations, such models are driven by white noise forces. Both the stochastic GOY and Sabra shell models have the form

$$
d u_{n}+\left(v k_{n}^{2} u_{n}+b_{n}(u, u)\right) d t=\sigma_{n} d \beta_{n}, \quad n=1,2, \ldots
$$

where $u_{n}(t)$ are complex valued, $v>0$ is a parameter that represents the viscosity, $k_{n}=k_{0} 2^{n}$ for some $k_{0}>0$ are representing wave numbers, $u(t)$ denotes the sequence $\left(u_{n}(t)\right)_{n \geq 1}$, $b_{n}(\cdot, \cdot)$ is a complex valued bilinear function of complex sequences $u=\left(u_{j}\right)_{j \geq 1}$, that depends only on the variables $u_{n-2}, u_{n-1}, u_{n+1}, u_{n+2}$ (where we impose the boundary conditions $\left.u_{-1}(t)=u_{0}(t)=0\right) . \sigma_{n}$ is a sequence of complex numbers, that are usually chosen equal to zero for all $n$ greater than some $n_{0}$ (which describes the range of wavenumbers and consequently the length scales of external forces), $\left(\beta_{n}\right)_{n \geq 1}$ is a sequence of independent complex valued Brownian motions. A rigorous theoretical analysis of the stochastic equation (1) and some of its statistical properties have been investigated in [4, 24], while other rigorous results in the case of deterministic force have been developed in [5, 12-14]. The exact form of $b_{n}(\cdot, \cdot)$ varies from one model to another. However, in all the various models in the sequel we assume that $b_{n}(\cdot, \cdot)$ is chosen in such a way that

$$
\sum_{n=1}^{\infty} b_{n}(u, v) \bar{v}_{n}=0,
$$

for all square summable sequences $u=\left(u_{m}\right)_{m \geq 1}$ and $v=\left(v_{m}\right)_{m \geq 1}$. Equation (2) implies a formal law of the conservation of energy in the inviscid $(v=0)$ and unforced form of (1).

In analogy with the statistical theory of turbulence it is interesting to investigate the accompanying linear advection equation to (1), that is the linear auxiliary equation in the unknown $w(t)=\left(w_{n}(t)\right)_{n \geq 1}$

$$
d w_{n}+\left(v k_{n}^{2} w_{n}+b_{n}(u, w)\right) d t=\sigma_{n} d \beta_{n}, \quad n=1,2, \ldots
$$

where $u$ is the solution of (1), and $w_{n}(t)$ are complex valued functions. There is an extensive literature investigating the statistical properties of linear advection (passive-scalar) equations in turbulent flows, which we do not pretend to cover in this contribution. We observe, however, that (3) is not the linearized version of (1) about the solution $u$. This is because the term $b_{n}(w, u)$ is missing from (3), and an additive force still appears in the right-hand side of (3). Equation (3) should be considered as an auxiliary equation which, to some extent, may have 
similar statistical properties to those of (1), but is amenable to linear analysis (for instance the use of propagators). There is some numerical and heuristic evidence that some statistical properties of the solutions to (3), like the scaling exponents of the structure functions, are the same as those of the solutions to (1), see [1] and [5]. It is then of interest to understand the properties of the joint system

$$
\begin{gathered}
d u_{n}+\left(v k_{n}^{2} u_{n}+b_{n}(u, u)\right) d t=\sigma_{n} d \beta_{n} \\
d w_{n}+\left(v k_{n}^{2} w_{n}+b_{n}(u, w)\right) d t=\sigma_{n} d \beta_{n},
\end{gathered}
$$

for $n=1,2, \ldots$ In addition, the following idea has been introduced first in [1] and proved rigorously later in [5]: one can symmetrize system (4) by means of two additional terms as follows

$$
\begin{gathered}
d u_{n}+\left(v k_{n}^{2} u_{n}+b_{n}(u, u)+\lambda b_{n}(w, u)\right) d t=\sigma_{n} d \beta_{n} \\
d w_{n}+\left(v k_{n}^{2} w_{n}+b_{n}(u, w)+\lambda b_{n}(w, w)\right) d t=\sigma_{n} d \beta_{n},
\end{gathered}
$$

where $\lambda \in \mathbb{R}$ is a parameter, and to analyze the dependence on $\lambda$ of the properties of (5). For $\lambda=0$ we recover (4). Observe that for $\lambda \neq 0$, setting $v=\lambda w$ and multiplying the second equation by $\lambda$, we have a perfectly symmetric system for the pair $(u, v)$, except for the force and initial conditions. Thus, to some extent, we would expect that $u$ and $\lambda w$ have similar statistical properties for $\lambda \neq 0$. If we consider, for instance, the structure function $S_{p}\left(k_{n}\right)=\left\langle\left|u_{n}\right|^{p}\right\rangle$ (we do not specify at this heuristic level the meaning of the averaging procedure $(\rangle$.$) , one might expect, as in the case of turbulent flows, that$

$$
S_{p}\left(k_{n}\right) \sim k_{n}^{-\zeta p}
$$

for $n$ lies in the so-called inertial range. The numbers $\zeta_{p}$ are called scaling exponents, which are universal in turbulent flows as the Reynolds number tends to infinity, i.e., as the viscosity tends to zero. Therefore, one possible definition of $\zeta_{p}$ is

$$
\lim _{\left(\nu, k_{n}\right) \rightarrow(0, \infty)} \frac{\log S_{p}\left(k_{n}\right)}{\log k_{n}}=-\zeta_{p},
$$

where the limit is taken along a region of the form $v^{\alpha} \leq k_{n}^{-1} \leq v^{\beta}$ for some $\alpha>0$ (usually $\alpha=\frac{4}{3}$ ). This is in order to ensure that the wavenumber considered are lying in the heart of the inertial range, as the viscosity tends to zero. Such statistical property, if it holds for $w$, it holds as well for $\lambda w$ with the same value $\zeta_{p}$ (and vice versa): indeed, if $S_{p}^{(w)}\left(k_{n}\right)$ and $S_{p}^{(\lambda w)}\left(k_{n}\right)$ are the structure functions of $w$ and $\lambda w$, respectively, we have $S_{p}^{(\lambda w)}\left(k_{n}\right)=\lambda^{p} S_{p}^{(w)}\left(k_{n}\right)$ and $\lim _{\left(v, k_{n}\right) \rightarrow(0, \infty)} \frac{\log \lambda^{p}}{\log k_{n}}=0$, which imply the claim. Thus, if the scaling exponents $\zeta_{p}$ exist for both $u$ and $\lambda w$ (this assumption seems to be reasonable based on numerical finding in $[1,5]$ and the references therein) and are equal (which is reasonable to assume thanks to the symmetry $u \leftrightarrow \lambda w$ described above), then they are equal for $u$ and $w$. In summary, it is, therefore, reasonable to expect that some statistical properties like the existence and the value of scaling exponents, are the same for $u$ and $w$, whenever $\lambda \neq 0$.

Finally, it will be of great interest to show that such statistical properties depend continuously on $\lambda$, as $\lambda \rightarrow 0$ : if this is true, then the solutions of (1) and (3) have the same statistical properties of the kind just described above. In particular, if this program is true, one is sure that results for the simpler linear model (3) can be translated to (1), which will be a remarkable breakthrough. 


\subsection{Content of the Paper}

The program above, outlined in [1] and [5], is composed of several steps, some of them are not easy to be justified rigorously. The first rigorous result has been obtained in [5] states that: in the case of deterministic forces, solutions of (5) depend continuously on $\lambda$ in $C([0, T] ; H \times H)$, for every given $T>0$. Here $H$ is the space of square summable sequences $\left(v_{n}\right)_{n \geq 1}$ in $\mathbb{C}$. This implies that the structure functions, defined as time average on any fixed finite time interval $[0, T]$ :

$$
S_{p}\left(k_{n}\right)=\frac{1}{T} \int_{0}^{T}\left(\left|u_{n}(t)\right|^{p}\right) d t
$$

depend continuously on $\lambda$. One of the limitations of this result of [5] that it considers deterministic forces. Here, we remove this restriction and prove the same result in the case described above of white noise forces.

Several other issues have to be solved in order to be able to claim that the program described above is complete. One of the other major issues in [5] is that the statistics is being considered on finite intervals of time $[0, T]$ instead of being considered on the attractor, i.e. as $T \rightarrow \infty$. The existence of the limit as $T \rightarrow \infty$, in the time average (definition of $S_{p}\left(k_{n}\right)$ ) of the deterministically forced system is, therefore, one of these issues of [5]. We do not directly address this difficult problem here, in the stochastically forced case, but we content ourselves with a structural result about the infinite time horizon properties of (5): we prove existence of a finite-dimensional random attractor. This is a pathwise property, in the vein of the property of continuous dependence on $\lambda$ in $C([0, T] ; H)$ stated above for the deterministic case. We hope that this result, or the techniques involved in establishing it, may contribute to the understanding of the problem of the long-term behavior, i.e. $T \rightarrow \infty$. Notice that we construct the random attractor for system (5) for every $\lambda \in \mathbb{R}$, hence if we take in particular $\lambda=0$ the first component of the system is decoupled and thus the projection of the attractor on the first component is the random attractor of equation (1). Thus we prove in this paper the existence of a finite-dimensional random attractor for the stochastic GOY and Sabra shell models, as a particular case of a more general result. However, the general result for system (5) may help to prove further results on the relations between the statistics of the nonlinear and the linear cases.

Due to the Itô nature of the previous equations, it is clear that other kind of analysis could be performed, in distribution and average sense instead of pathwise. This will be done elsewhere. We restrict ourselves here to purely pathwise properties.

The paper is organized as follows. In Sect. 2, we present the functional framework and prove pathwise well-posedness of system (5), and the continuous dependence of the solutions on $\lambda$. In Sect. 3, we give some preliminary results about random attractors and some of their properties. In Sect. 4, we prove the existence of a random attractor for every coefficient $\lambda \in \mathbb{R}$, its upper semicontinuity with respect to $\lambda$; and finally that the random attractor has a finite Hausdorff dimension.

\section{Well-Posedness and Continuous Dependence on $\lambda$}

\subsection{Functional Setting}

Let us introduce the following spaces of complex valued sequences; we consider them as vector spaces on the field of real numbers. The space $H$ is the space of $l^{2}$ sequences over 
the field of complex numbers $\mathbb{C}$ :

$$
H=\left\{u=\left(u_{n}\right)_{n \geq 1}: u_{n} \in \mathbb{C} \text { for all } n \geq 1 \text { and } \sum_{n=1}^{\infty}\left|u_{n}\right|^{2}<\infty\right\} .
$$

It is a Hilbert space with the inner product $\langle u, v\rangle_{H}:=\operatorname{Re} \sum_{n=1}^{\infty} u_{n} \bar{v}_{n}$ and the norm given by $|u|_{H}^{2}=\sum_{n=1}^{\infty}\left|u_{n}\right|^{2}$. Let us recall that we have defined $k_{n}=2^{n} k_{0}, n \geq 1$, with $k_{0}>0$ given. We introduce now the Hilbert spaces $D(A) \subset V \subset H$ defined as

$$
V=\left\{u \in H: \sum_{n=1}^{\infty} k_{n}^{2}\left|u_{n}\right|^{2}<\infty\right\}
$$

with norm $\|u\|_{V}^{2}=\sum_{n=1}^{\infty} k_{n}^{2}\left|u_{n}\right|^{2}$. Moreover, for all $\alpha \geq 0$, we define

$$
D\left(A^{\alpha}\right)=\left\{u \in H: \sum_{n=1}^{\infty} k_{n}^{4 \alpha}\left|u_{n}\right|^{2}<\infty\right\} .
$$

On the latter space we define the linear operator $A^{\alpha}: D\left(A^{\alpha}\right) \subset H \rightarrow H$ as

$$
\left(A^{\alpha} u\right)_{n}=k_{n}^{2 \alpha} u_{n}, \quad \text { for all } \quad u \in D\left(A^{\alpha}\right) .
$$

The operator $A^{\alpha}$ is self-adjoint and strictly positive definite:

$$
\left\langle A^{\alpha} u, u\right\rangle_{H} \geq k_{0}^{2 \alpha}|u|_{H}^{2}, \quad \text { for all } \quad u \in D\left(A^{\alpha}\right) .
$$

We also observe that the inclusion maps of $D(A) \subset V$ and $V \subset H$ are compact embeddings. We finally introduce the bilinear operator $B(\cdot, \cdot): V \times V \rightarrow H$. For the GOY shell model it is defined as

$$
\begin{aligned}
b_{n}(u, v) & :=(B(u, v))_{n} \\
& :=i k_{n}\left(\frac{1}{4} \bar{v}_{n-1} \bar{u}_{n+1}-\frac{1}{2}\left(\bar{u}_{n+1} \bar{v}_{n+2}+\bar{v}_{n+1} \bar{u}_{n+2}\right)+\frac{1}{8} \bar{u}_{n-1} \bar{v}_{n-2}\right) .
\end{aligned}
$$

For the Sabra shell model we define it as

$$
\begin{aligned}
b_{n}(u, v):=(B(u, v))_{n}:= & \frac{i}{3} k_{n+1}\left[(1+\delta) \bar{v}_{n+1} u_{n+2}+(2-\delta) \bar{u}_{n+1} v_{n+2}\right] \\
& +\frac{i}{3} k_{n}\left[(1-2 \delta) \bar{u}_{n-1} v_{n+1}-(1+\delta) \bar{v}_{n-1} u_{n+1}\right] \\
& +\frac{i}{3} k_{n-1}\left[(2-\delta) u_{n-1} v_{n-2}+(1-2 \delta) u_{n-2} v_{n-1}\right]
\end{aligned}
$$

(see [12-14]), where $\delta$ is a real number. In both shell models we impose the boundary conditions $u_{-1}=u_{0}=0$. What distinguishes the Sabra shell model from the GOY one is the dependence of the former on the parameter $\delta$, which is in charge for changing its character from the so-called $2 \mathrm{~d}$ Turbulence regime to the $3 \mathrm{~d}$ Turbulence regime, depending on the definiteness of the sign of a second (in addition to the energy) quadratic conserved quantity; see $[5,12-14]$ and [32]. 
For both the GOY and Sabra shell models, the operator $B(\cdot, \cdot)$ is a bilinear continuous operator from $V \times H$ to $H$, and also from $H \times V$ to $H$, as it will be stated in the next lemma. We also state its basic skew-symmetry property.

Lemma 1 There is a constant $C>0$ such that

$$
|B(u, v)|_{H} \leq C\|u\|_{V}|v|_{H}, \quad u \in V, v \in H
$$

and

$$
|B(u, v)|_{H} \leq C\|v\|_{V}|u|_{H}, \quad v \in V, u \in H .
$$

Hence, $B(\cdot, \cdot)$ is a bilinear continuous operator from $V \times H$ to $H$, and from $H \times V$ to $H$. Moreover,

$$
\langle B(u, v), v\rangle_{H}=0
$$

for all $u \in V$ and $v \in H$, or $v \in V$ and $u \in H$.

Proof The first inequality follows from the fact that

$$
\sum_{n=1}^{\infty} k_{n}^{2}\left|u_{n}\right|^{2}\left|v_{n}\right|^{2} \leq\left(\sup _{n} k_{n}^{2}\left|u_{n}\right|^{2}\right) \sum_{n=1}^{\infty}\left|v_{n}\right|^{2} \leq\|u\|_{V}^{2}|v|_{H}^{2}
$$

and the second inequality follows similarly by interchanging $u$ and $v$. Having proved these facts, the expression in the last identity is well defined. The proof of $\sum_{n=1}^{\infty} \operatorname{Re}\left[B(u, v)_{n} \bar{v}_{n}\right]=$ 0 can be found in [4] for the Goy model and in [12] for the Sabra model.

We will also consider the space $V^{\prime}$, the dual space of $V$, which can be identified as

$$
V^{\prime}=\left\{u=\left(u_{n}\right)_{n \geq 1}: u_{n} \in \mathbb{C} \text { for all } n \geq 1, \text { and } \sum_{n=1}^{\infty} k_{n}^{-2}\left|u_{n}\right|^{2}<\infty\right\}
$$

with the norm $|u|_{V^{\prime}}^{2}:=\sum_{n=1}^{\infty} k_{n}^{-2}\left|u_{n}\right|^{2}, u \in V^{\prime}$. It is clear that $H \subset V^{\prime}$, and $V^{\prime}$ is the dual of $V$ (with respect to $H$ ), with dual pairing between $V^{\prime}$ and $V$ defined as

$$
\langle u, v\rangle_{V^{\prime}, V}:=\operatorname{Re} \sum_{n=1}^{\infty} u_{n} \bar{v}_{n}, \quad \forall u \in V^{\prime}, v \in V .
$$

Observe that $\langle u, v\rangle_{H}=\langle u, v\rangle_{V^{\prime}, V}$, when $u \in H$, for every $v \in V$.

It is easy to extend the operator $A$ as a bounded linear operator from $V$ to $V^{\prime}$. One can also extend $B$ to a bilinear operator $B(\cdot, \cdot): H \times H \rightarrow V^{\prime}$. The definition is possible because

$$
\begin{aligned}
|B(u, v)|_{V^{\prime}}^{2} & =\sum_{n=1}^{\infty} k_{n}^{-2}\left|B(u, v)_{n}\right|^{2} \leq C_{*}\left(\sum_{n=1}^{\infty}\left|v_{n}\right|^{2}\right)\left(\sup _{n \geq 1}\left|u_{n}\right|^{2}\right) \\
& \leq C_{*}\left(\sum_{n=1}^{\infty}\left|v_{n}\right|^{2}\right)\left(\sum_{n=1}^{\infty}\left|u_{n}\right|^{2}\right)=C_{*}|u|_{H}^{2}|v|_{H}^{2} .
\end{aligned}
$$


We also have $\langle B(u, v), z\rangle_{V^{\prime}, V}=-\langle B(u, z), v\rangle_{H}$ for all $u, v \in H, z \in V$ by Lemma 1 and a density argument.

Define $\tilde{H}=H \times H, \tilde{V}=V \times V$ and $D\left(\tilde{A}^{\alpha}\right)=D\left(A^{\alpha}\right) \times D\left(A^{\alpha}\right)$, for $\alpha \geq 0$. If $x=$ $\left(x_{1}, x_{1}\right) \in \tilde{H}$ and $y=\left(y_{1}, y_{2}\right) \in \tilde{H}$, we define the scalar product in $\tilde{H}$ as

$$
\langle x, y\rangle_{\tilde{H}}=\left\langle x_{1}, y_{1}\right\rangle_{H}+\left\langle x_{2}, y_{2}\right\rangle_{H}
$$

and the norms in $\tilde{H}$ and $\tilde{V}$ as

$$
\begin{gathered}
|x|_{\tilde{H}}^{2}=\left|x_{1}\right|_{H}^{2}+\left|x_{2}\right|_{H}^{2}, \quad x=\left(x_{1}, x_{2}\right) \in \tilde{H} \\
\|x\|_{\tilde{V}}^{2}=\left\|x_{1}\right\|_{V}^{2}+\left\|x_{2}\right\|_{V}^{2}, \quad x=\left(x_{1}, x_{2}\right) \in \tilde{V} .
\end{gathered}
$$

Moreover, define the linear operator $\tilde{A}: D(\tilde{A}) \subset \tilde{H} \rightarrow \tilde{H}$, or also $\tilde{A}: \tilde{V} \rightarrow \tilde{V}^{\prime}$, as $\tilde{A} x=$ $\left(A x_{1}, A x_{2}\right)$ and, for every $\lambda \in \mathbb{R}$, define the bilinear continuous operator $\tilde{B}_{\lambda}$ from $\tilde{V} \times \tilde{H}$ to $\tilde{H}$ or from $\tilde{H} \times \tilde{V}$ to $\tilde{H}$ as

$$
\tilde{B}_{\lambda}(x, y)=\left(B\left(x_{1}, y_{1}\right)+\lambda B\left(x_{2}, y_{1}\right), B\left(x_{1}, y_{2}\right)+\lambda B\left(x_{2}, y_{2}\right)\right),
$$

where as usual we have used the notation $x=\left(x_{1}, x_{2}\right), y=\left(y_{1}, y_{2}\right)$. The main properties of the operator $\tilde{B}_{\lambda}$ are listed in the following lemma, whose proof is an easy consequence of Lemma 1.

Lemma 2 There is a constant $C>0$ such that

$$
\left|\tilde{B}_{\lambda}(u, v)\right|_{\tilde{H}} \leq C\|u\|_{\tilde{V}}|v|_{\tilde{H}}, \quad \text { for every } \quad u \in \tilde{V}, v \in \tilde{H},
$$

and

$$
\left|\tilde{B}_{\lambda}(u, v)\right|_{\tilde{H}} \leq C\|v\|_{\tilde{V}}|u|_{\tilde{H}}, \quad \text { for every } \quad v \in \tilde{V}, u \in \tilde{H}
$$

Moreover,

$$
\left\langle\tilde{B}_{\lambda}(u, v), v\right\rangle_{\tilde{H}}=0 \quad \text { for all } u \in \tilde{V}, v \in \tilde{H} \quad \text { or } \quad v \in \tilde{V}, u \in \tilde{H} .
$$

2.2 Well-Posedness, Stochastic Flow and Pathwise Version in $\lambda$

Let $\left(\sigma_{n}\right)$ be a sequence of complex numbers such that

$$
\sum k_{n}^{2 \varepsilon}\left|\sigma_{n}\right|^{2}<\infty
$$

for some $\varepsilon>0$. This is a standing assumption for the sequel.

Let $\Omega$ be the space of continuous functions from $\mathbb{R}$ to $H$, null at zero, endowed with the metric of uniform convergence on compact sets. Let $\mathcal{F}$ be the Borel $\sigma$-field associated with $\Omega$. Denote by $(W(t))_{t \in \mathbb{R}}$ the canonical process defined on $\Omega$ as $W(t, \omega)=\omega(t)$, for every $\omega \in \Omega$. Let $P$ be a probability measure on $(\Omega, \mathcal{F})$ such that $(W(t))_{t \geq 0}$ and $(W(-t))_{t \geq 0}$ are $P$-a.s. two independent Brownian motions in $H$ with the same covariance. We call $P$ a two sided Wiener probability measure and $(W(t))_{t \in \mathbb{R}}$ a two sided Brownian motion. Such objects exist, for every given covariance operator, and play an important role in the theory of random dynamical systems, see [3]. Details on infinite-dimensional Brownian motions and 
their stochastic integration can be found in [19]. We will also denote by $E$ the expectation on $(\Omega, \mathcal{F}, P)$.

For simplicity of the computations, and in analogy with equation (1), we assume that the components $\left(W_{n}(t)\right)_{t \in \mathbb{R}}$, for all $n \geq 1$, of the two-sided Brownian motion have the form

$$
W_{n}(t)=\sigma_{n} \beta_{n}(t)
$$

where $\beta_{n}(t)$ are independent two-sided complex Brownian motions on $(\Omega, \mathcal{F}, P)$ (with incremental covariance equal to one) and $\left(\sigma_{n}\right)$ is the sequence given above.

On the probability space $(\Omega, \mathcal{F}, P)$ consider the family of transformations $\left\{\theta_{t}: \Omega \mapsto\right.$ $\Omega, t \in \mathbb{R}\}$ defined as $\theta_{t} \omega=\omega(t+\cdot)-\omega(t)$, for every $\omega \in \Omega$. They are measure preserving and ergodic with respect to $P$, and satisfy $\theta_{0}=I d, \theta_{t+s}=\theta_{t} \circ \theta_{s}$, for $s, t \in \mathbb{R}$, see [3].

Let $\left(\mathcal{F}_{t}\right)_{t \in \mathbb{R}}$ be the filtration associated to $(W(t))_{t \in \mathbb{R}}\left(\mathcal{F}_{t}\right.$ is generated by $W(s)$ for all $s \leq t)$.

Given initial conditions $u_{0}, w_{0} \in H$, let us first rewrite system (5) in the abstract form

$$
\left\{\begin{array}{l}
d u^{\lambda}=\left[-v A u^{\lambda}-B\left(u^{\lambda}, u^{\lambda}\right)-\lambda B\left(w^{\lambda}, u^{\lambda}\right)\right] d t+d W \\
d w^{\lambda}=\left[-v A w^{\lambda}-B\left(u^{\lambda}, w^{\lambda}\right)-\lambda B\left(w^{\lambda}, w^{\lambda}\right)\right] d t+d W \\
u^{\lambda}(0)=u_{0} \\
w^{\lambda}(0)=w_{0} .
\end{array}\right.
$$

We consider the above Cauchy problem on $[0, \infty)$. Using the notation of the previous section, system (8) can be rewritten as follows

$$
d \tilde{u}^{\lambda}+\left(v \tilde{A} \tilde{u}^{\lambda}+\tilde{B}_{\lambda}\left(\tilde{u}^{\lambda}, \tilde{u}^{\lambda}\right)\right) d t=d \tilde{W}, \quad \tilde{u}^{\lambda}(0)=\tilde{u}_{0}
$$

where $\tilde{u}^{\lambda}(t)=\left(u^{\lambda}(t), w^{\lambda}(t)\right), \tilde{u}_{0}=\left(u_{0}, w_{0}\right), \tilde{W}(t)=(W(t), W(t))$. As an introductory step, let us first give the usual definition of solution of (9); however, we will eventually need a more refined notion of solution, that we will introduce in Definition 4 below.

Definition 3 Given $\tilde{u}_{0} \in \tilde{H}$, we say that a stochastic process $\tilde{u}^{\lambda}(t, \omega)$ is a solution of (9) if it is a continuous adapted process in $\tilde{H}$ on $\left(\Omega, \mathcal{F},\left(\mathcal{F}_{t}\right)_{t \geq 0}, P\right)$ and, for $P$-a.e. $\omega \in \Omega$,

$$
\begin{aligned}
& \tilde{u}^{\lambda}(\cdot, \omega) \in C([0, T] ; \tilde{H}) \cap L^{2}(0, T ; \tilde{V}) \quad \text { for all } T>0 \\
& \left\langle\tilde{u}^{\lambda}(t, \omega), \psi\right\rangle_{\tilde{H}}+\int_{0}^{t} v\left\langle\tilde{u}^{\lambda}(s, \omega), \tilde{A} \psi\right\rangle_{\tilde{V}, \tilde{V}^{\prime}} d s \\
& \quad+\int_{0}^{t}\left\langle\tilde{B}_{\lambda}\left(\tilde{u}^{\lambda}(s, \omega), \tilde{u}^{\lambda}(s, \omega)\right), \psi\right\rangle_{\tilde{H}} d s=\left\langle\tilde{u}_{0}, \psi\right\rangle_{\tilde{H}}+\langle\tilde{W}(t, \omega), \psi\rangle_{\tilde{H}}
\end{aligned}
$$

for $t \geq$ and $\psi \in \tilde{V}$.

Notice that $\tilde{u}^{\lambda}(s, \omega) \in \tilde{V}$ for a.e. $s \geq 0$, hence the integral of the bilinear term is well defined.

The above definition is sufficient to analyze individual solutions, but the theory of random attractors requires the concept of stochastic flow: the $P$-negligible set where the properties of the above definition may not hold, that is, it must be independent of $\tilde{u}_{0}$, and for $P$ a.e. $\omega \in \Omega$, moreover, we will also need continuity with respect to the initial value $\tilde{u}_{0}$. In 
addition, here, we want to "vary" the parameter $\lambda$ independently of $\omega$ : a priori this is not possible, again because the $P$-negligible set where the properties of the previous definition hold, may depend on $\lambda$. Both problems can be solved because it is possible to perform a complete pathwise analysis of the equation. Let us, therefore, give a more appropriate definition of solution for (9), which is relevant to the above mentioned issues.

Definition 4 A stochastic flow depending on $\lambda \in \mathbb{R}$, associated with (9), is a family of mappings $\left\{\varphi^{\lambda}(t, \omega): \tilde{H} \rightarrow \tilde{H} ; t \geq 0, \omega \in \Omega^{0}, \lambda \in \mathbb{R}\right\}$, where $\Omega^{0} \in \mathcal{F}$ is $\theta_{t}$-invariant and $P\left(\Omega^{0}\right)=1$, with the properties:

1. for every $\lambda \in \mathbb{R}$ and $\tilde{u}_{0} \in \tilde{H},(t, \omega) \mapsto \varphi^{\lambda}(t, \omega) \tilde{u}_{0}$ (arbitrarily extended to all $\omega \in \Omega$ ) is a continuous adapted process in $\tilde{H}$ on $\left(\Omega, \mathcal{F},\left(\mathcal{F}_{t}\right)_{t \geq 0}, P\right)$ and for every $\omega \in \Omega^{0}$ we have

$$
\varphi^{\lambda}(\cdot, \omega) \tilde{u}_{0} \in C([0, T] ; \tilde{H}) \cap L^{2}(0, T ; \tilde{V}), \quad \text { for all } T>0,
$$

and

$$
\begin{aligned}
& \left\langle\varphi^{\lambda}(t, \omega) \tilde{u}_{0}, \psi\right\rangle_{\tilde{H}}+\int_{0}^{t} v\left\langle\varphi^{\lambda}(s, \omega) \tilde{u}_{0}, \tilde{A} \psi\right\rangle_{\tilde{V}, \tilde{V}^{\prime}} d s \\
& \quad+\int_{0}^{t}\left\langle\tilde{B}_{\lambda}\left(\varphi^{\lambda}(s, \omega) \tilde{u}_{0}, \varphi^{\lambda}(s, \omega) \tilde{u}_{0}\right), \psi\right\rangle_{\tilde{H}} d s=\left\langle\tilde{u}_{0}, \psi\right\rangle_{\tilde{H}}+\langle\tilde{W}(t, \omega), \psi\rangle_{\tilde{H}}
\end{aligned}
$$

for $t \geq 0$ and $\psi \in \tilde{V}$;

2. for every $\lambda \in \mathbb{R}$ and $\omega \in \Omega^{0}, \varphi^{\lambda}(t, \omega)$ is a continuous map from $\tilde{H}$ into itself, for all $t \geq 0 ;$ and

$$
\varphi^{\lambda}(t+s, \omega)=\varphi^{\lambda}\left(t, \theta_{s} \omega\right) \circ \varphi^{\lambda}(s, \omega) \text { for all } t, s \geq 0 .
$$

To emphasize the role of $\Omega^{0}$ in Definition 4 , we will consider stochastic flows depending on $\lambda \in \mathbb{R}$, defined on the set $\Omega^{0}$.

We have the following result. The concept of uniqueness of stochastic flow depending on $\lambda$ means: if we have two stochastic flows, defined on two sets $\Omega_{1}^{0}$ and $\Omega_{2}^{0}$, then they coincide on a set $\Omega_{3}^{0} \in \mathcal{F}$ such that $P\left(\Omega_{3}^{0}\right)=1$.

Theorem 5 Under assumption (7), there exists a unique stochastic flow depending on $\lambda \in \mathbb{R}$, in the sense of Definition 4, associated with (9).

\section{Proof}

Step 1 (preliminary facts). Denote by $e^{-v \tilde{A} t}$ the analytic semigroup generated by $\tilde{A}$ (see, e.g., [33]). By the general theory of analytic semigroups or by explicit computation based on the spectral representation, for every $\alpha>0$ we have $\left|\tilde{A}^{\alpha} e^{-v \tilde{A} t}\right|_{\tilde{H}} \leq \frac{C_{\alpha}}{t^{\alpha}}$ for some constant $C_{\alpha}>0$. Moreover, notice that $\|x\|_{\tilde{V}}=\left\|\tilde{A}^{1 / 2} x\right\|_{\tilde{H}}$ for all $x \in \tilde{V}$.

The process $\tilde{A}^{\varepsilon / 2} \tilde{W}(t)$ has $H$-components $\left(A^{\varepsilon / 2} W(t), A^{\varepsilon / 2} W(t)\right)$ where $A^{\varepsilon / 2} W(t)$ has complex components $k_{n}^{\varepsilon} \sigma_{n} \beta_{n}(t)$. Thanks to assumption (7) it follows that $\tilde{A}^{\varepsilon / 2} \tilde{W}(t)$ is an $\tilde{H}$-valued Brownian motion. Thus it is $\gamma$-Hölder continuous, with respect to $t$, in $\tilde{H}$, for every exponent $\gamma<\frac{1}{2}$, see [19]. This means that there exists a set $\Omega_{W}^{0} \in \mathcal{F}$ such that $P\left(\Omega_{W}^{0}\right)=1$ and $\tilde{A}^{\varepsilon / 2} \tilde{W}(t, \omega)$ is $\gamma$-Hölder continuous for every exponent $\gamma<\frac{1}{2}$, for every 
$\omega \in \Omega_{W}^{0}$. The set $\Omega_{W}^{0}$ is $\theta_{t}$-invariant, because Hölder continuity is preserved by translation.

Step 2 (auxiliary Stokes type problem). The pathwise analysis of (9) requires a careful analysis of an auxiliary process. The process we are going to introduce is usually defined as

$$
\tilde{z}(t)=\int_{0}^{t} e^{-v \tilde{A}(t-s)} d \tilde{W}(s)
$$

but from this definition via a stochastic integral (which is a $P$-equivalence class) it is less easy to justify the $\theta_{t}$-invariance of certain properties, on a full measure set $\Omega^{0}$. For this reason we adopt the following less intuitive definition. See [23] for further details on this approach.

Let $\omega \in \Omega_{W}^{0}$ be given throughout this step, where $\Omega_{W}^{0}$ has been defined in step 1 . The function $t \mapsto \tilde{z}(t, \omega)$ given by

$$
\tilde{z}(t, \omega)=e^{-v \tilde{A} t} \tilde{W}(t, \omega)+\int_{0}^{t} \nu \tilde{A} e^{-v \tilde{A}(t-s)}(\tilde{W}(t, \omega)-\tilde{W}(s, \omega)) d s,
$$

is well defined and bounded in $\tilde{V}$, because (for $\varepsilon$ that is given in assumption (7)) we have

$$
\begin{aligned}
\left\|e^{-\nu \tilde{A} t} \tilde{W}(t, \omega)\right\|_{\tilde{V}} & =\left|\tilde{A}^{1 / 2-\varepsilon / 2} e^{-\nu \tilde{A} t} \tilde{A}^{\varepsilon / 2}(\tilde{W}(t, \omega)-\tilde{W}(0, \omega))\right|_{\tilde{H}} \\
& \leq\left|\tilde{A}^{1 / 2-\varepsilon / 2} e^{-\nu \tilde{A} t}\right|_{\tilde{H}}\left|\tilde{A}^{\varepsilon / 2}(\tilde{W}(t, \omega)-\tilde{W}(0, \omega))\right|_{\tilde{H}} \\
& \leq C \frac{1}{t^{1 / 2-\varepsilon / 2}} t^{\beta},
\end{aligned}
$$

for every $\beta<\frac{1}{2}$ and a suitable constant $C>0$ that depends on $\beta$ and $\omega$. Observe that in the last estimate we used the details described in step 1 above, in particular, the Hölder continuity. Similarly

$$
\begin{aligned}
& \left\|\int_{0}^{t} \tilde{A} e^{-v \tilde{A}(t-s)}(\tilde{W}(t, \omega)-\tilde{W}(s, \omega)) d s\right\|_{\tilde{V}} \\
& \quad \leq \int_{0}^{t}\left|\tilde{A}^{3 / 2-\varepsilon / 2} e^{-v \tilde{A}(t-s)} \tilde{A}^{\varepsilon / 2}(\tilde{W}(t, \omega)-\tilde{W}(s, \omega))\right|_{\tilde{H}} d s \\
& \quad \leq \int_{0}^{t} C \frac{1}{(t-s)^{3 / 2-\varepsilon / 2}}(t-s)^{\beta} d s .
\end{aligned}
$$

The above estimates imply that $\|\tilde{z}(t, \omega)\|_{\tilde{V}}$ is bounded on the interval $[0, T]$, for all $T>0$ given, and the bound depends on $T$ and $\omega$.

It is easy to deduce that

$$
\langle\tilde{z}(t, \omega), \psi\rangle_{\tilde{H}}+\int_{0}^{t} v\langle\tilde{z}(s, \omega), \tilde{A} \psi\rangle_{\tilde{V}, \tilde{V}^{\prime}} d s=\langle\tilde{W}(t, \omega), \psi\rangle_{\tilde{H}}
$$

for all $t \geq 0$ and $\psi \in \tilde{V}$. 
Step 3 (auxiliary Navier-Stokes type random equation). Let $\omega \in \Omega_{W}^{0}$ be given, and let $\tilde{z}(t, \omega))$ satisfy $(10)$ or $(11)$. Let us introduce the auxiliary random differential equation

$$
\frac{d \tilde{v}^{\lambda}(t, \omega)}{d t}+v \tilde{A} \tilde{v}^{\lambda}(t, \omega)+\tilde{B}_{\lambda}\left(\tilde{v}^{\lambda}(t, \omega)+\tilde{z}(t, \omega), \tilde{v}^{\lambda}(t, \omega)+\tilde{z}(t, \omega)\right)=0
$$

for $t \geq 0$ and $\tilde{v}^{\lambda}(0, \omega)=\tilde{u}_{0}$.

For every $\omega \in \Omega_{W}^{0}$ and $\lambda \in \mathbb{R}$, there exists a unique weak solution $\tilde{v}^{\lambda}(\cdot, \omega)=\tilde{v}^{\lambda}\left(\cdot, \omega, \tilde{u}_{0}\right)$ of (12) (the definition of weak solutions is similar to Definition 4) and it depends continuously, in $C([0, T] ; \tilde{H}) \cap L^{2}(0, T ; \tilde{V})$ norms, for any given $T>0$, on the initial condition $\tilde{u}_{0}$ in $\tilde{H}$. A full rigorous proof of this statement is very long, but at the same time it is very classical. Similar detailed proofs are given, for instance in [4, 22], and in [11] or [34] in the case of the classical Navier-Stokes equations (i.e., when $\tilde{z}=0$ ). The rigorous detailed proof is based on the Galerkin approximation procedure and then passing to the limit using the appropriate compactness theorems. These classical computations can be found in the above references. Moreover, similar computations will be performed in the second part of the paper for the existence of the random attractor.

Step 4 (existence of the stochastic flow). For every $\lambda \in \mathbb{R}, t \geq 0, \omega \in \Omega_{W}^{0}$ and $\tilde{u}_{0} \in \tilde{H}$, define

$$
\varphi^{\lambda}(t, \omega) \tilde{u}_{0}=\tilde{v}^{\lambda}\left(t, \omega, \tilde{u}_{0}\right)+\tilde{z}(t, \omega)
$$

where $\tilde{v}^{\lambda}\left(\cdot, \omega, \tilde{u}_{0}\right)$ is the unique weak solution given in step 3 and $\tilde{z}(\cdot, \omega)$ is defined in step 2. The set $\Omega_{W}^{0}$ is $\theta_{t}$-invariant and $P\left(\Omega_{W}^{0}\right)=1$. Property 1 of Definition 4 is a direct consequence of the analogous properties of $\tilde{v}^{\lambda}\left(\cdot, \omega, \tilde{u}_{0}\right)$ and $\tilde{z}(\cdot, \omega)$ proved in steps 2 and 3. As to property 2 of Definition 4 , given $\lambda \in \mathbb{R}, \omega \in \Omega_{W}^{0}, t \geq 0$, the continuity of $\varphi^{\lambda}(t, \omega)$ in $\tilde{H}$ is a consequence of the continuous dependence of $\tilde{v}^{\lambda}\left(\cdot, \omega, \tilde{u}_{0}\right)$ on $\tilde{u}_{0}$, see step 3 . The property

$$
\varphi^{\lambda}(t+s, \omega) \tilde{u}_{0}=\varphi^{\lambda}\left(t, \theta_{s} \omega\right) \varphi^{\lambda}(s, \omega) \tilde{u}_{0}
$$

for all $t, s \geq 0$ follows from the uniqueness statement of step 3 and the following property of the process $\tilde{W}(t, \omega)=(W(t, \omega), W(t, \omega)), W(t, \omega)=\omega(t)$,

$$
W\left(t, \theta_{s} \omega\right)=\theta_{s} \omega(t)=\omega(s+t)-\omega(s)=W(s+t, \omega)-W(s, \omega) .
$$

Step 5 (uniqueness of the stochastic flow). Let $\varphi_{1}^{\lambda}(t, \omega)$ be a stochastic flow depending on $\lambda \in \mathbb{R}$, associated with (9), defined on a $\theta_{t}$-invariant full measure set $\Omega_{1}^{0}$. Consider the $\theta_{t^{-}}$ invariant full measure set $\Omega_{W}^{0}$ described in step 2 above. The set $\Omega_{1}^{0} \cap \Omega_{W}^{0}$ is $\theta_{t}$-invariant and $P\left(\Omega_{1}^{0} \cap \Omega_{W}^{0}\right)=1$. For all $\omega \in \Omega_{1}^{0} \cap \Omega_{W}^{0}$ define

$$
\tilde{v}_{1}^{\lambda}\left(t, \omega, \tilde{u}_{0}\right):=\varphi_{1}^{\lambda}(t, \omega) \tilde{u}_{0}-\tilde{z}(t, \omega) .
$$

From the properties of $\varphi_{1}^{\lambda}$ and $\tilde{z}$ it is trivial to check that $\tilde{v}_{1}^{\lambda}\left(\cdot, \omega, \tilde{u}_{0}\right)$ is a weak solution of the auxiliary equation of step 3 . Of course we have $\varphi_{1}^{\lambda}(t, \omega) \tilde{u}_{0}=\tilde{v}_{1}^{\lambda}\left(t, \omega, \tilde{u}_{0}\right)+\tilde{z}(t, \omega)$. Now, let $\varphi_{2}^{\lambda}(t, \omega)$ be another stochastic flow depending on $\lambda \in \mathbb{R}$, associated with (9), with its $\theta_{t}$-invariant full measure set $\Omega_{2}^{0}$. The function

$$
\tilde{v}_{2}^{\lambda}\left(t, \omega, \tilde{u}_{0}\right):=\varphi_{2}^{\lambda}(t, \omega) \tilde{u}_{0}-\tilde{z}(t, \omega)
$$


defined for $\omega \in \Omega_{2}^{0} \cap \Omega_{W}^{0}$ is a weak solution of the auxiliary equation of step 3. Thus, for $\omega \in$ $\Omega_{1}^{0} \cap \Omega_{2}^{0} \cap \Omega_{W}^{0}$ we have $\tilde{v}_{1}^{\lambda}\left(t, \omega, \tilde{u}_{0}\right)=\tilde{v}_{2}^{\lambda}\left(t, \omega, \tilde{u}_{0}\right)$ because of the uniqueness of solutions for (12), for every $\omega \in \Omega_{W}^{0}$. Hence, $\varphi_{1}^{\lambda}(t, \omega) \tilde{u}_{0}=\varphi_{2}^{\lambda}(t, \omega) \tilde{u}_{0}$ for all $\omega \in \Omega_{1}^{0} \cap \Omega_{2}^{0} \cap \Omega_{W}^{0}$. Since $P\left(\Omega_{1}^{0} \cap \Omega_{2}^{0} \cap \Omega_{W}^{0}\right)=1$, the proof is complete.

In step 5 of the previous proof we have obtained also the following representation result, which will be useful in the next section.

Corollary 6 Let $\varphi^{\lambda}(t, \omega)$ be a stochastic flow depending on $\lambda \in \mathbb{R}$, associated with (9), defined on a $\theta_{t}$-invariant full measure set $\Omega_{1}^{0}$. On the $\theta_{t}$-invariant full measure set $\Omega_{W}^{0}$ described in step 2 of Theorem 5 , one can define the functions $\tilde{z}(t, \omega)$ and $\tilde{v}^{\lambda}\left(t, \omega, \tilde{u}_{0}\right)$ according to steps 2 and 3 of that proof. Then, on the $\theta_{t}$-invariant full measure set $\Omega^{0}:=\Omega_{1}^{0} \cap \Omega_{W}^{0}$ we have

$$
\varphi^{\lambda}(t, \omega) \tilde{u}_{0}=\tilde{v}^{\lambda}\left(t, \omega, \tilde{u}_{0}\right)+\tilde{z}(t, \omega)
$$

2.3 Continuous Dependence with Respect to the Parameter $\lambda$

As above, we assume condition (7). The uniformity in the initial condition of the next statement will be used to prove the upper semicontinuity of the random attractor with respect to the parameter $\lambda$.

Theorem 7 Let $\varphi^{\lambda}(t, \omega)$ be the stochastic flow that was established in Theorem 5 and Corollary 6 , associated with (9) and depending on the parameter $\lambda \in \mathbb{R}$. Let $\Omega^{0} \in \mathcal{F}, P\left(\Omega^{0}\right)=1$, be a $\theta_{t}$-invariant set where all the properties of Definition 4 and Corollary 6 hold true. Then, for every $\omega \in \Omega^{0}$, we have

$$
\lim _{\lambda \rightarrow \lambda_{0}} \sup _{\tilde{u}_{0} \in B} \sup _{0 \leq t \leq T}\left|\varphi^{\lambda}(t, \omega) \tilde{u}_{0}-\varphi^{\lambda_{0}}(t, \omega) \tilde{u}_{0}\right|_{\tilde{H}}=0
$$

for all $T>0, \lambda_{0} \in \mathbb{R}$ and all bounded sets $B \subset \tilde{H}$.

Proof We prove the theorem only in the case $\lambda_{0}=0$, the general case being the same. The elements $\omega \in \Omega^{0}$ and $T>0$ are given and fixed throughout the proof, as well as the bounded set $B \subset \tilde{H}$.

Step 1 (preparation). Denote by $\left(u^{\lambda}\left(t, \omega, \tilde{u}_{0}\right), w^{\lambda}\left(t, \omega, \tilde{u}_{0}\right)\right)$ the decomposition of $\varphi^{\lambda}(t, \omega) \tilde{u}_{0}$ in $\tilde{H}=H \times H$, and by $\left(u_{0}, w_{0}\right)$ the decomposition of the initial value $\tilde{u}_{0}$. Where it is necessary, we will shorten the notation and write $\left(u^{\lambda}(t), w^{\lambda}(t)\right)$ and apply analogous change of notation to other similar quantities.

From the weak integral equation in Definition 4 and the definitions of $\tilde{A}$ and $\tilde{B}_{\lambda}$ we have

$$
\begin{aligned}
& \left\langle u^{\lambda}(t), \psi_{1}\right\rangle_{H}+\int_{0}^{t} v\left\langle u^{\lambda}(s), A \psi_{1}\right\rangle_{V, V^{\prime}} d s \\
& \quad+\int_{0}^{t}\left\langle B\left(u^{\lambda}(s), u^{\lambda}(s)\right)+\lambda B\left(w^{\lambda}(s), u^{\lambda}(s)\right), \psi_{1}\right\rangle_{H} d s \\
& =\left\langle u_{0}, \psi_{1}\right\rangle_{H}+\left\langle W(t, \omega), \psi_{1}\right\rangle_{H},
\end{aligned}
$$


for all $t \geq 0$ and $\psi_{1} \in V$ and

$$
\begin{aligned}
& \left\langle w^{\lambda}(t), \psi_{2}\right\rangle_{H}+\int_{0}^{t} v\left\langle w^{\lambda}(s), A \psi_{2}\right\rangle_{V, V^{\prime}} d s \\
& \quad+\int_{0}^{t}\left\langle B\left(u^{\lambda}(s), w^{\lambda}(s)\right)+\lambda B\left(w^{\lambda}(s), w^{\lambda}(s)\right), \psi_{2}\right\rangle_{H} d s \\
& =\left\langle w_{0}, \psi_{2}\right\rangle_{H}+\left\langle W(t, \omega), \psi_{2}\right\rangle_{H},
\end{aligned}
$$

for all $t \geq 0$ and $\psi_{2} \in V$. Let us define the new function

$$
q^{\lambda}\left(t, \omega, \tilde{u}_{0}\right):=u^{\lambda}\left(t, \omega, \tilde{u}_{0}\right)+\lambda w^{\lambda}\left(t, \omega, \tilde{u}_{0}\right)
$$

and the corresponding difference

$$
\rho^{\lambda}\left(t, \omega, \tilde{u}_{0}\right):=q^{\lambda}\left(t, \omega, \tilde{u}_{0}\right)-q^{0}\left(t, \omega, \tilde{u}_{0}\right)=q^{\lambda}\left(t, \omega, \tilde{u}_{0}\right)-u^{0}\left(t, \omega, \tilde{u}_{0}\right) .
$$

The above quantities are solutions, respectively, of

$$
\begin{aligned}
& \left\langle q^{\lambda}(t), \psi_{1}\right\rangle_{H}+\int_{0}^{t} v\left\langle q^{\lambda}(s), A \psi_{1}\right\rangle_{V, V^{\prime}} d s+\int_{0}^{t}\left\langle B\left(q^{\lambda}(s), q^{\lambda}(s)\right), \psi_{1}\right\rangle_{H} d s \\
& =\left\langle u_{0}+\lambda w_{0}, \psi_{1}\right\rangle_{H}+(1+\lambda)\left\langle W(t, \omega), \psi_{1}\right\rangle_{H},
\end{aligned}
$$

for $t \geq 0$ and $\psi_{1} \in V$, and

$$
\begin{aligned}
& \left\langle\rho^{\lambda}(t), \psi_{2}\right\rangle_{H}+\int_{0}^{t} v\left\langle\rho^{\lambda}(s), A \psi_{2}\right\rangle_{V, V^{\prime}} d s \\
& \quad+\int_{0}^{t}\left\langle B\left(q^{\lambda}(s), \rho^{\lambda}(s)\right)+B\left(\rho^{\lambda}(s), q^{\lambda}(s)\right)-B\left(\rho^{\lambda}(s), \rho^{\lambda}(s)\right), \psi_{2}\right\rangle_{H} d s \\
& =\left\langle\lambda w_{0}, \psi_{2}\right\rangle_{H}+\lambda\left\langle W(t, \omega), \psi_{2}\right\rangle_{H},
\end{aligned}
$$

for $t \geq 0$ and $\psi_{2} \in V$.

Step 2 (bound on $q^{\lambda}$ ). Let us prove next that

$$
\sup _{\lambda \in[-1,1]} \sup _{\tilde{u}_{0} \in B} \sup _{0 \leq t \leq T}\left|q^{\lambda}\left(t, \omega, \tilde{u}_{0}\right)\right|_{H}<\infty .
$$

Define

$$
\bar{v}^{\lambda}\left(t, \omega, \tilde{u}_{0}\right):=q^{\lambda}\left(t, \omega, \tilde{u}_{0}\right)-(1+\lambda) z(t, \omega),
$$

where $z(t, \omega)$ is any one of the two equal components of $\tilde{z}(t, \omega)$ given in Corollary 6 . We have

$$
\begin{aligned}
& \left\langle\bar{v}^{\lambda}(t), \psi_{1}\right\rangle_{H}+\int_{0}^{t} v\left\langle\bar{v}^{\lambda}(s), A \psi_{1}\right\rangle_{V, V^{\prime}} d s \\
& \quad+\int_{0}^{t}\left\langle B\left(\bar{v}^{\lambda}(s)+(1+\lambda) z(s), \bar{v}^{\lambda}(s)+(1+\lambda) z(s)\right), \psi_{1}\right\rangle_{H} d s \\
& =\left\langle u_{0}+\lambda w_{0}, \psi_{1}\right\rangle_{H},
\end{aligned}
$$


for all $t \geq 0$ and $\psi_{1} \in V$. Formally, this implies

$$
\frac{1}{2} \frac{d}{d t}\left|\bar{v}^{\lambda}\right|_{H}^{2}+v\left\|\bar{v}^{\lambda}\right\|_{V}^{2} \leq \mid\left\langle B\left(\bar{v}^{\lambda}+(1+\lambda) z, \bar{v}^{\lambda}+(1+\lambda) z\right),\left.\bar{v}^{\lambda}\right|_{H}\right|
$$

and thus

$$
\begin{aligned}
& \frac{1}{2}\left|\bar{v}^{\lambda}(t)\right|_{H}^{2}+v \int_{0}^{t}\left\|\bar{v}^{\lambda}(s)\right\|_{V}^{2} d s \leq \frac{1}{2}\left|u_{0}+\lambda w_{0}\right|_{H}^{2} \\
& \quad+\int_{0}^{t}\left|\left\langle B\left(\bar{v}^{\lambda}(s)+(1+\lambda) z(s), \bar{v}^{\lambda}(s)+(1+\lambda) z(s)\right), \bar{v}^{\lambda}(s)\right\rangle_{H}\right| d s
\end{aligned}
$$

Rigorously, the above inequality can be proved either by general abstract theorems (see [34]) or by taking finite-dimensional (i.e. with finite many components) test functions $\psi_{1}$, performing the computations at the finite dimensional level and then taking the limit, which can be justified because the map

$$
s \mapsto\left\langle B\left(\bar{v}^{\lambda}(s)+(1+\lambda) z(s), \bar{v}^{\lambda}(s)+(1+\lambda) z(s)\right), \bar{v}^{\lambda}(s)\right\rangle_{H}
$$

is integrable. Thus, from Lemma 2 and the bounds on $z(t, \omega)$, given in step 2 of the proof of Theorem 5, we have, for $t \in[0, T]$,

$$
\begin{aligned}
& \frac{1}{2}\left|\bar{v}^{\lambda}(t)\right|_{H}^{2}+v \int_{0}^{t}\left\|\bar{v}^{\lambda}(s)\right\|_{V}^{2} d s-\frac{1}{2}\left|u_{0}+\lambda w_{0}\right|_{H}^{2} \\
& \quad \leq 2 \int_{0}^{t} \mid\left\langle B\left(\bar{v}^{\lambda}(s)+(1+\lambda) z(s), z(s)\right),\left.\bar{v}^{\lambda}(s)\right|_{H}\right| d s \\
& \quad \leq C \int_{0}^{t}\left|\bar{v}^{\lambda}(s)\right|_{H}\left|\bar{v}^{\lambda}(s)+(1+\lambda) z(s)\right|_{H}\|z(s)\|_{V} d s \\
& \leq C(\omega) \int_{0}^{t}\left|\bar{v}^{\lambda}(s)\right|_{H}\left(\left|\bar{v}^{\lambda}(s)\right|_{H}+C(\omega)\right) d s,
\end{aligned}
$$

where $C(\omega)$ depends on the bounds of the relevant norms of $z(\cdot, \omega)$ over the interval $[0, T]$, which in principle is also depending on $T$. By Gronwall lemma we deduce

$$
\left|\bar{v}^{\lambda}\left(t, \omega, \tilde{u}_{0}\right)\right|_{H} \leq C(\omega) \cdot\left|\bar{v}^{\lambda}\left(0, \omega, \tilde{u}_{0}\right)\right|_{H}
$$

on $[0, T]$, for a new constant $C(\omega)$. This implies

$$
\left|q^{\lambda}\left(t, \omega, \tilde{u}_{0}\right)\right|_{H} \leq 2|z(t, \omega)|_{H}+C(\omega) \cdot\left|u_{0}+\lambda w_{0}\right|_{H},
$$

on $[0, T]$, and the claim of this step is proved, using again the bounds on $z(\cdot, \omega)$ over the interval $[0, T]$.

Step 3 (convergence of $\rho^{\lambda}$ ). Next we prove that

$$
\lim _{\lambda \rightarrow 0} \sup _{\tilde{u}_{0} \in B} \sup _{0 \leq t \leq T}\left|\rho^{\lambda}\left(t, \omega, \tilde{u}_{0}\right)\right|_{H}=0 .
$$


In one sentence, this is a consequence of the various bounds, that we have established previously, and the fact that the initial condition $\lambda w_{0}$ and the forcing term $\lambda W(t, \omega)$ converge to zero, as $\lambda \rightarrow 0$. Define

$$
\hat{v}^{\lambda}\left(t, \omega, \tilde{u}_{0}\right):=\rho^{\lambda}\left(t, \omega, \tilde{u}_{0}\right)-\lambda z(t, \omega),
$$

it satisfies

$$
\begin{aligned}
& \left\langle\hat{v}^{\lambda}(t), \psi_{2}\right\rangle_{H}+\int_{0}^{t} v\left\langle\hat{v}^{\lambda}(s), A \psi_{2}\right\rangle_{V, V^{\prime}} d s \\
& \quad+\int_{0}^{t}\left\langle B\left(q^{\lambda}(s), \rho^{\lambda}(s)\right)+B\left(\rho^{\lambda}(s), q^{\lambda}(s)\right)-B\left(\rho^{\lambda}(s), \rho^{\lambda}(s)\right), \psi_{2}\right\rangle_{H} d s \\
& =\left\langle\lambda w_{0}, \psi_{2}\right\rangle_{H},
\end{aligned}
$$

for all $t \geq 0$ and $\psi_{2} \in V$. By virtue of Lemmas 1 and 2, we have

$$
\left|\left\langle B\left(q^{\lambda}, \rho^{\lambda}\right), \hat{v}^{\lambda}\right\rangle_{H}\right|=\mid\left\langle B\left(q^{\lambda}, \lambda z\right),\left.\left.\hat{v}^{\lambda}\right|_{H}|\leq C| \lambda\left|\|z\|_{V}\right| q^{\lambda}\right|_{H}\left|\hat{v}^{\lambda}\right|_{H} .\right.
$$

Thus, using both the bound on $z(\cdot, \omega)$ on $[0, T]$ and the bound of the previous step, there is constant $C_{1}(\omega)$ such that

$$
\left|\left\langle B\left(q^{\lambda}, \rho^{\lambda}\right), \hat{v}^{\lambda}\right\rangle_{H}\right| \leq \lambda^{2} C_{1}(\omega)+\left|\hat{v}^{\lambda}\right|_{H}^{2},
$$

for all $\tilde{u}_{0} \in B$ and $t \in[0, T]$. Similarly,

$$
\begin{aligned}
\left|\left\langle B\left(\rho^{\lambda}, q^{\lambda}\right), \hat{v}^{\lambda}\right\rangle_{H}\right| & =\left|\left\langle B\left(\hat{v}^{\lambda}+\lambda z, \hat{v}^{\lambda}\right), q^{\lambda}\right\rangle_{H}\right| \leq C\left\|\hat{v}^{\lambda}\right\|_{V}\left|q^{\lambda}\right|_{H}\left(\left|\hat{v}^{\lambda}\right|_{H}+|\lambda||z|_{H}\right) \\
& \leq \frac{v}{2}\left\|\hat{v}^{\lambda}\right\|_{V}^{2}+C_{2}(\omega)\left|\hat{v}^{\lambda}\right|_{H}^{2}+\frac{\lambda^{2}}{v} C_{2}(\omega),
\end{aligned}
$$

for some constant $C_{2}(\omega)$, and

$$
\begin{aligned}
& \mid\left\langle B\left(\rho^{\lambda}, \rho^{\lambda}\right),\left.\hat{v}^{\lambda}\right|_{H}\right| \\
& \quad=\left|\left\langle B\left(\hat{v}^{\lambda}+\lambda z, \lambda z\right), \hat{v}^{\lambda}\right\rangle_{H}\right| \leq C|\lambda|\|z\|_{V}\left|\hat{v}^{\lambda}\right|_{H}\left(\left|\hat{v}^{\lambda}\right|_{H}+|\lambda||z|_{H}\right) \\
& \quad \leq|\lambda| C_{3}(\omega)\left|\hat{v}^{\lambda}\right|_{H}\left(\left|\hat{v}^{\lambda}\right|_{H}+|\lambda| C_{3}(\omega)\right) \leq|\lambda| C_{3}(\omega)\left|\hat{v}^{\lambda}\right|_{H}^{2}+|\lambda|^{3} C_{3}(\omega),
\end{aligned}
$$

for some constant $C_{3}(\omega)$. Hence, from the equation in weak form for $\hat{v}^{\lambda}$ (and similarly to the proof of step 2 above) we deduce, for $\lambda \in[-1,1]$,

$$
\begin{aligned}
& \frac{1}{2}\left|\hat{v}^{\lambda}(t)\right|_{H}^{2}+\frac{v}{2} \int_{0}^{t}\left\|\hat{v}^{\lambda}(s)\right\|_{V}^{2} d s \\
& \quad \leq \frac{1}{2}\left|\lambda w_{0}\right|_{H}^{2}+\int_{0}^{t}\left(C_{4}(\omega)\left|\hat{v}^{\lambda}(s)\right|_{H}^{2}+\lambda^{2}\left(1+\frac{1}{v}\right) C_{4}(\omega)\right) d s
\end{aligned}
$$

for some constant $C_{4}(\omega)$. By Gronwall lemma we get

$$
\lim _{\lambda \rightarrow 0} \sup _{\tilde{u}_{0} \in B} \sup _{0 \leq t \leq T}\left|\hat{v}^{\lambda}\left(t, \omega, \tilde{u}_{0}\right)\right|_{H}=0,
$$

which implies the claim of this step. 
Step 4 (convergence of $w^{\lambda}$ ). With the notation $\xi^{\lambda}\left(t, \omega, \tilde{u}_{0}\right):=w^{\lambda}\left(t, \omega, \tilde{u}_{0}\right)-w^{0}\left(t, \omega, \tilde{u}_{0}\right)$, let us prove that

$$
\lim _{\lambda \rightarrow 0} \sup _{\tilde{u}_{0} \in B} \sup _{0 \leq t \leq T}\left|\xi^{\lambda}\left(t, \omega, \tilde{u}_{0}\right)\right|_{H}=0 .
$$

We have

$$
\begin{gathered}
\left\langle\xi^{\lambda}(t), \psi\right\rangle_{H}+\int_{0}^{t} v\left\langle\xi^{\lambda}(s), A \psi\right\rangle_{V, V^{\prime}} d s=\int_{0}^{t}\left\langle B\left(u^{0}(s), w^{0}(s)\right), \psi\right\rangle_{H} d s \\
-\int_{0}^{t}\left\langle B\left(u^{\lambda}(s), w^{\lambda}(s)\right)+\lambda B\left(w^{\lambda}(s), w^{\lambda}(s)\right), \psi\right\rangle_{H} d s \\
=\int_{0}^{t}\left\langle B\left(q^{0}(s), w^{0}(s)\right), \psi\right\rangle_{H} d s-\int_{0}^{t}\left\langle B\left(q^{\lambda}(s), w^{\lambda}(s)\right), \psi\right\rangle_{H} d s \\
=-\int_{0}^{t}\left\langle B\left(\rho^{\lambda}(s), w^{0}(s)\right), \psi\right\rangle_{H} d s-\int_{0}^{t}\left\langle B\left(q^{\lambda}(s), \xi^{\lambda}(s)\right), \psi\right\rangle_{H} d s,
\end{gathered}
$$

for all $t \geq 0$ and $\psi \in V$. As in the previous steps we deduce that

$$
\begin{aligned}
& \frac{1}{2}\left|\xi^{\lambda}(t)\right|_{H}^{2}+v \int_{0}^{t}\left\|\xi^{\lambda}(s)\right\|_{V}^{2} d s \\
& \quad \leq \int_{0}^{t}\left|\left\langle B\left(\rho^{\lambda}(s), w^{0}(s)\right), \xi^{\lambda}(s)\right\rangle_{H}\right| d s+\int_{0}^{t} \mid\left\langle B\left(q^{\lambda}(s), \xi^{\lambda}(s)\right),\left.\xi^{\lambda}(s)\right|_{H}\right| d s \\
& \quad \leq C \int_{0}^{t}\left|w^{0}(s)\right|_{H}\left\|\xi^{\lambda}(s)\right\|_{V}\left|\rho^{\lambda}(s)\right|_{H} d s
\end{aligned}
$$

Hence,

$$
\frac{1}{2}\left|\xi^{\lambda}(t)\right|_{H}^{2}+\frac{v}{2} \int_{0}^{t}\left\|\xi^{\lambda}(s)\right\|_{V}^{2} d s \leq \frac{C}{v} \int_{0}^{t}\left|w^{0}(s)\right|_{H}^{2}\left|\rho^{\lambda}(s)\right|_{H}^{2} d s .
$$

This implies the claim of this step.

Step 5 (convergence of $u^{\lambda}$ ). We simply notice that

$$
\left|u^{\lambda}(t)-u^{0}(t)\right|_{H}=\left|q^{\lambda}(t)-q^{0}(t)-\lambda w^{\lambda}(t)\right|_{H},
$$

therefore, by the results of steps 3 and 4 , we have

$$
\lim _{\lambda \rightarrow 0} \sup _{\tilde{u}_{0} \in B} \sup _{0 \leq t \leq T}\left|u^{\lambda}\left(t, \omega, \tilde{u}_{0}\right)-u^{0}\left(t, \omega, \tilde{u}_{0}\right)\right|_{H}=0 .
$$

The proof is complete.

\section{Random Dynamical Systems}

In this section we recall few definitions from the theory of random dynamical systems. For general notions and results see [3], and see [9] for analogous concept for non-autonomous dynamical systems. Here, we mainly refer to specific notions from [18]. Similar notions are also given in [17]. 


\subsection{The Basic Set-up}

Let $(X, d)$ be a Polish space. Recall that the mapping $\varphi$ of Definition 4 is called a random dynamical system (RDS) on $X$ over $\left(\Omega, \mathcal{F}, P, \theta_{t}\right)$.

Definition 8 A closed random set $K$ is said to absorb the set $B \subset X, B$ is fixed non-random, if there exists a random variable $t_{B}(\omega)$ such that, for $P$-a.e. $\omega \in \Omega$,

$$
\varphi\left(t, \theta_{-t} \omega\right) B \subset K(\omega) \text { for all } t>t_{B}(\omega)
$$

The smallest $t_{B}(\omega) \geq 0$ for which (14) holds is called the random absorption time of $B$ by $K$.

Remark 9 Note that $\varphi\left(t, \theta_{-t} \omega\right) x$ can be thought of as the position of the trajectory at time 0 , which was in $x$ at time $-t$.

Definition 10 A random set $\mathcal{A}(\omega)$ is called a random attractor associated with the random dynamical system $\varphi$ if, for $P$-a.e. $\omega \in \Omega$, the following is satisfied:

1. $\mathcal{A}(\omega)$ is a nonempty compact subset of $X$,

2. $\varphi(t, \omega) \mathcal{A}(\omega)=\mathcal{A}\left(\theta_{t} \omega\right), \forall t \geq 0$,

3. for every $B \subset X$ bounded (and non-random)

$$
\lim _{t \rightarrow \infty} d_{H}\left(\varphi\left(t, \theta_{-t} \omega\right) B, \mathcal{A}(\omega)\right)=0
$$

The following theorem about the existence of random attractors is due to Crauel and Flandoli [18].

Theorem 11 Suppose there exists a closed random set $D$ which is absorbing every bounded non-random set $B \subset X$, and for which $D(\omega)$ is a compact subset of $X$ for $P$-a.e. $\omega \in \Omega$. Then, there exists a random attractor for $\varphi$.

Remark 12 In Crauel [16] it is shown that, under the ergodicity assumption on $\theta_{t}$, there exists a compact set $K(\omega) \subset X$ such that, for $P$-a.e. $\omega \in \Omega$, the random attractor is the $\omega$-limit set of $K(\omega)$, that is,

$$
\mathcal{A}(\omega)=\bigcap_{n \geq 0} \overline{\bigcup_{t \geq n} \varphi\left(t, \theta_{-t} \omega\right) K(\omega)}
$$

\subsection{Random Attractor Dimensionality}

We are interested in the property of finite Hausdorff dimensionality of the random attractor. The two most relevant results for this purpose are the works of Debussche [20,21]. We apply the result of the second one of these papers, based on a property called random squeezing property, which was inspired by the squeezing property in the deterministic case that was introduced in [26] (see, also, [15] and [31]). This property is also used in the proof of finite number of determining modes see for e.g., $[6,25]$. The fact that the random attractor is not uniformly bounded makes the corresponding random squeezing property depend exponentially on a random variable. However, an ergodic argument will make it possible to work with this weaker property. 
Definition 13 [20] Let $H$ be a separable Hilbert space with norm $|\cdot|_{H}, \varphi(t, \omega)$ a random dynamical system in $H$ with random attractor $\mathcal{A}(\omega)$. We say that $\varphi(t, \omega)$ satisfies the random squeezing property if there exist a random variable $C_{5}(\omega)$, a finite-dimensional projector $\Pi$ in $H$, and positive numbers $\mu, \delta$ such that, for $P$-a.e. $\omega \in \Omega$,

$$
\left|\Pi \varphi(t, \omega) u_{0}-\Pi \varphi(t, \omega) v_{0}\right|_{H} \leq e^{\int_{0}^{t} C_{5}\left(\theta_{s} \omega\right) d s}\left|u_{0}-v_{0}\right|_{H}
$$

and

$$
\left|(I-\Pi)\left(\varphi(t, \omega) u_{0}-\varphi(t, \omega) v_{0}\right)\right|_{H} \leq\left(e^{-\mu t}+\delta e^{\int_{0}^{t} C_{5}\left(\theta_{s} \omega\right) d s}\right)\left|u_{0}-v_{0}\right|_{H}
$$

for every $t \geq 0$, and every $u_{0}, v_{0} \in \mathcal{A}(\omega)$.

Theorem 14 [20] There exist absolute constants $K_{1}, K_{2}, K_{3}$ such that if $\varphi(t, \omega)$ is a random dynamical system that satisfies:

(i) the random squeezing property, mentioned in Definition 13, with a random variable $C_{5}(\omega)$, a finite-dimensional projector $\Pi$ and two positive numbers $\mu, \delta$, and

(ii) the expected value with respect to the measure $P$

$$
E\left(C_{5}(\omega)\right)<\infty, \quad \delta \leq K_{1} \quad \text { and } \quad \mu \geq K_{2} E\left(C_{5}(\omega)\right),
$$

then, for $P$-a.s. $\omega \in \Omega$, the random attractor $\mathcal{A}(\omega)$ of $\varphi(t, \omega)$ has finite Hausdorff dimension which is less than $K_{3} R(\Pi) \log R(\Pi)$, where $R(\Pi)$ is the rank of the projector $\Pi$.

\section{Application to the Shell Model}

In Theorem 5 we have constructed, for every $\lambda \in \mathbb{R}$, a random dynamical system $\varphi^{\lambda}(t, \omega)$ associated with (9). In this section we prove the existence of the random attractor associated with $\varphi^{\lambda}(t, \omega)$. At the end of the section we also prove that the random attractor, as a function of $\lambda$, is upper semi-continuous. For the upper semi-continuity of deterministic attractors with respect to a parameter see, e.g., [30].

\subsection{Auxiliary Problem}

As in step 2 of Sect. 2.2, we will introduce an auxiliary Ornstein-Uhlenbeck process. This is a slightly different process but will have mainly the same properties as the process introduced in step 2 of Sect. 2.2. Following the steps of Sect. 2, let $\Omega_{W}^{0} \in \mathcal{F}$ introduced in Sect. 2. Let $\alpha>0$ be an arbitrary constant. For $\omega \in \Omega_{W}^{0}$, let

$$
\tilde{z}^{\alpha}(t, \omega)=\int_{-\infty}^{t}(\nu \tilde{A}+\alpha I) e^{-(\nu \tilde{A}+\alpha I)(t-s)}(\tilde{W}(t, \omega)-\tilde{W}(s, \omega)) d s
$$

which is well defined and bounded in $\tilde{V}$.

The process $\tilde{z}^{\alpha}(t), t \in \mathbb{R}$ is a Gaussian, stationary and ergodic process. Moreover, it is a solution of the equation

$$
d \tilde{z}^{\alpha}(t)=(-v \tilde{A}-\alpha) \tilde{z}^{\alpha}(t) d t+d \tilde{W}(t),
$$


i.e. for all $t \in \mathbb{R}$ and $P$-a.s.

$$
\tilde{z}^{\alpha}(t)=\int_{-\infty}^{t} e^{-(v \tilde{A}+\alpha)(t-s)} d \tilde{W}(s)
$$

Moreover,

$$
E\left\|\tilde{z}^{\alpha}(t)\right\|_{\tilde{V}}^{2}=E\left|\tilde{A}^{1 / 2} \tilde{z}^{\alpha}(t)\right|_{\tilde{H}}^{2}=E \sum_{n=1}^{\infty}\left(k_{n} \tilde{z}_{n}^{\alpha}(t)\right)^{2}=\sum_{n=1}^{\infty} \frac{k_{n}^{2} \sigma_{n}^{2}}{2\left(\nu k_{n}^{2}+\alpha\right)}<\infty,
$$

by using the isometry formula for the Itô integral.

Furthermore, $E\left\|\tilde{z}^{\alpha}(t)\right\|_{\tilde{V}}^{2}$ tends to 0 , when $\alpha \rightarrow \infty$. In particular, there exists $\alpha_{*}(v)>0$ such that

$$
E\left\|\tilde{z}^{\alpha}(t)\right\|_{\tilde{V}}^{2} \leq \frac{k_{0} v}{8 C_{*}} \quad \text { for all } \quad \alpha \geq \alpha_{*},
$$

where $C_{*}$ is the constant in the inequality (6).

\subsection{Absorbing Compact Set}

Let $\omega \in \Omega_{W}^{0}$ be given and let us introduce the random differential equation

$$
\frac{d \tilde{v}(t, \omega)}{d t}+v \tilde{A} \tilde{v}(t, \omega)=-\tilde{B}_{\lambda}\left(\tilde{v}(t, \omega)+\tilde{z}^{\alpha}(t, \omega), \tilde{v}(t, \omega)+\tilde{z}^{\alpha}(t, \omega)\right)+\alpha \tilde{z}^{\alpha}(t, \omega)
$$

Notice that in fact $\tilde{v}$ depends on $\alpha$, because $\tilde{z}^{\alpha}$ depends on $\alpha$. It is not difficult to prove, using a Galerkin method, that for each $\omega \in \Omega_{W}^{0}$ and $t_{0} \in \mathbb{R}$, with $\tilde{v}_{t_{0}}(\omega) \in \tilde{H}$ is given, there exists a unique solution $\tilde{v}(t, \omega)$ defined on $\left[t_{0}, \infty\right)$ to (22) such that

$$
\tilde{v}\left(t_{0}, \omega\right)=\tilde{v}_{t_{0}}(\omega),
$$

and such that $\tilde{v}(\cdot, \omega) \in C\left(\left[t_{0}, \infty\right), \tilde{H}\right) \bigcap L^{2}\left(\left(t_{0}, \infty\right), \tilde{V}\right)$. We refer to [22] for more detailed computations.

Let us define $\varphi^{\lambda}(t, \omega) \tilde{u}_{t_{0}}=\tilde{v}(t, \omega)+\tilde{z}^{\alpha}(t, \omega)$, where $\tilde{v}$ is the solution of (22) with $\tilde{v}_{t_{0}}(\omega)=\tilde{u}_{t_{0}}(\omega)-\tilde{z}^{\alpha}\left(t_{0}, \omega\right)$.

We would like to prove the existence of a compact absorbing set in $\tilde{H}$ at time $t=0$. Through this section we will take $B$ to be a bounded set in $\tilde{H}$, and that for any $t_{0} \in \mathbb{R}$ we will assume $\tilde{u}\left(t_{0}\right) \in B$; moreover, $\tilde{v}$ is the solution of (22) and (23) with $\tilde{v}_{t_{0}}(\omega)=\tilde{u}\left(t_{0}, \omega\right)-$ $\tilde{z}^{\alpha}\left(t_{0}, \omega\right)$.

Let $t_{0}<-1$ and $t \in[-1,0]$.

Lemma 15 Let $\tilde{v}_{t_{0}} \in \tilde{H}$ and $\tilde{v}$ be a solution of (22) associated with the initial condition $\tilde{v}_{t_{0}}$. Then, for all $t_{0}<-1$ and for all $t \in[-1,0]$

$$
|\tilde{v}(t)|_{\tilde{H}}^{2} \leq\left|\tilde{v}\left(t_{0}\right)\right|_{\tilde{H}}^{2} e^{\int_{t_{0}}^{t}\left(2 C_{*}\left\|\tilde{z}^{\alpha}(s)\right\|_{\tilde{V}}-\frac{k_{0} v}{2}\right) d s}+\int_{t_{0}}^{t} f(s) e^{\int_{s}^{t}\left(2 C_{*}\left\|\tilde{z}^{\alpha}(r)\right\|_{\tilde{V}}-\frac{k_{0} v}{2}\right) d r} d s
$$


where

$$
f(t):=\frac{4 C_{*}^{2}}{v}\left|\tilde{z}^{\alpha}(t)\right|_{\tilde{H}}^{4}+\frac{8 \alpha^{2}}{k_{0} v}\left|\tilde{z}^{\alpha}(t)\right|_{\tilde{H}}^{2}
$$

and $C_{*}$ is the constant in the inequality (6).

Proof As before, the proof is formal and can be made rigorous by applying the Galerkin approximation procedure. Let us take the inner produce in $\tilde{H}$ of (22) with $\tilde{v}$ to obtain

$$
\left\langle\frac{d \tilde{v}}{d t}, \tilde{v}\right\rangle+v\langle\tilde{A} \tilde{v}, \tilde{v}\rangle=-\left\langle\tilde{B}_{\lambda}\left(\tilde{v}+\tilde{z}^{\alpha}, \tilde{v}+\tilde{z}^{\alpha}\right), \tilde{v}\right\rangle+\alpha\left\langle\tilde{z}^{\alpha}, \tilde{v}\right\rangle .
$$

Using Lemma 2, inequality (6) and Young's inequality, we estimate the right-hand side of the above and get

$$
\begin{aligned}
\mid\left\langle\tilde{B}_{\lambda}\left(\tilde{v}+\tilde{z}^{\alpha}, \tilde{v}+\tilde{z}^{\alpha}, \tilde{v}\right\rangle\right| & \left.=\left|-\tilde{B}_{\lambda}(\tilde{v}, \tilde{v}), \tilde{z}^{\alpha}\right\rangle-\tilde{B}_{\lambda}\left(\tilde{z}^{\alpha}, \tilde{v}\right), \tilde{z}^{\alpha}\right\rangle \mid \\
& \leq\left\|\tilde{B}_{\lambda}(\tilde{v}, \tilde{v})\right\|_{\tilde{V}^{\prime}}\left\|\tilde{z}^{\alpha}\right\|_{\tilde{V}}+\left|\tilde{B}_{\lambda}\left(\tilde{z}^{\alpha}, \tilde{v}\right)\right|_{\tilde{H}}\left|\tilde{z}^{\alpha}\right|_{\tilde{H}} \\
& \leq C_{*}\left(|\tilde{v}|_{\tilde{H}}^{2}\left\|\tilde{z}^{\alpha}\right\|_{\tilde{V}}+\|\tilde{v}\|_{\tilde{V}}\left|\tilde{z}^{\alpha}\right|_{\tilde{H}}^{2}\right) \\
& \leq C_{*}|\tilde{v}|_{\tilde{H}}^{2}\left\|\tilde{z}^{\alpha}\right\|_{\tilde{V}}+\frac{v}{2}\|\tilde{v}\|_{\tilde{V}}^{2}+\frac{2 C_{*}^{2}}{v}\left|\tilde{z}^{\alpha}\right|_{\tilde{H}}^{4} .
\end{aligned}
$$

For the other term we apply the Cauchy-Schwarz and Young's inequalities we have

$$
\left|\alpha\left\langle\tilde{z}^{\alpha}, \tilde{v}\right\rangle\right| \leq \alpha\left|\tilde{z}^{\alpha}\right|_{\tilde{H}}|\tilde{v}|_{\tilde{H}} \leq \frac{k_{0} v}{4}|\tilde{v}|_{\tilde{H}}^{2}+\frac{4 \alpha^{2}}{k_{0} v}\left|\tilde{z}^{\alpha}\right|_{\tilde{H}}^{2}
$$

Hence, we get that

$$
\frac{1}{2} \frac{d}{d t}|\tilde{v}|_{\tilde{H}}^{2}+\frac{v}{2}\|\tilde{v}\|_{\tilde{V}}^{2} \leq C_{*}|\tilde{v}|_{\tilde{H}}^{2}\left\|\tilde{z}^{\alpha}\right\|_{\tilde{V}}+\frac{2 C_{*}^{2}}{v}\left|\tilde{z}^{\alpha}\right|_{\tilde{H}}^{4}+\frac{k_{0} v}{4}|\tilde{v}|_{\tilde{H}}^{2}+\frac{4 \alpha^{2}}{k_{0} v}\left|\tilde{z}^{\alpha}\right|_{\tilde{H}}^{2} .
$$

Using the Poincaré-like inequality $v\|\tilde{v}\|_{\tilde{V}}^{2} \geq v k_{0}|\tilde{v}|_{\tilde{H}}^{2}$, we get

$$
\frac{d}{d t}|\tilde{v}|_{\tilde{H}}^{2} \leq 2 C_{*}|\tilde{v}|_{\tilde{H}}^{2}\left\|\tilde{z}^{\alpha}\right\|_{\tilde{V}}+\frac{4 C_{*}^{2}}{v}\left|\tilde{z}^{\alpha}\right|_{\tilde{H}}^{4}-\frac{k_{0} v}{2}|\tilde{v}|_{\tilde{H}}^{2}+\frac{8 \alpha^{2}}{k_{0} v}\left|\tilde{z}^{\alpha}\right|_{\tilde{H}}^{2}
$$

We integrate over $\left(t_{0}, t\right)$ and get

$$
|\tilde{v}(t)|_{\tilde{H}}^{2} \leq\left|\tilde{v}\left(t_{0}\right)\right|_{\tilde{H}}^{2}+\int_{t_{0}}^{t}\left(2 C_{*}\left\|\tilde{z}^{\alpha}(r)\right\|_{\tilde{V}}-\frac{k_{0} v}{2}\right)|\tilde{v}(r)|_{\tilde{H}}^{2} d r+\int_{t_{0}}^{t} f(r) d r .
$$

Using Gronwall Lemma we get the result. 
Lemma 16 Let $\alpha \geq \alpha_{*}(v)$, such that (21) holds. Suppose that the assumptions of Lemma 15 are satisfied, and let $f$ be given by Lemma 15. Let

$$
R_{1}^{t}(\omega):=1+\int_{-\infty}^{t} f(s) e^{\int_{s}^{t}\left(2 C_{*}\left\|\tilde{z}^{\alpha}(r)\right\|_{\tilde{V}}-\frac{k_{0} v}{2}\right) d r} d s
$$

then, the ball $B\left(0, R_{1}^{0}(\omega)\right) \subset \tilde{H}$ is an absorbing set at time $t \in[-1,0]$, for the system (22).

Notice that in principle $R_{1}^{t}(\omega)$ depends on $\alpha$, however, if we fix $\alpha=\alpha_{0}:=2 \alpha_{*}(v)$ (see (21)) then $R_{1}^{t}(\omega)$ will depend only on $v$ and the statement will still be valid.

Proof Using the ergodicity of the process $\tilde{z}^{\alpha}$ we have that

$$
-\frac{1}{t_{0}} \int_{t_{0}}^{0}\left\|\tilde{z}^{\alpha}(s)\right\|_{\tilde{V}} d s \rightarrow_{t_{0} \rightarrow-\infty} E\left\|\tilde{z}^{\alpha}(0)\right\|_{\tilde{V}}, \quad P \text {-a.s. }
$$

Hence, there exists $s_{0}(\omega)<0$ such that for every $t_{0} \leq s_{0}(\omega)$,

$$
-\frac{1}{t_{0}} \int_{t_{0}}^{0}\left\|\tilde{z}^{\alpha}(s)\right\|_{\tilde{V}} d s \leq 2 E\left\|\tilde{z}^{\alpha}(0)\right\|_{\tilde{V}} .
$$

Then,

$$
\exp \left(t_{0}\left(\frac{k_{0} v}{2}+\frac{1}{t_{0}} \int_{t_{0}}^{0} 2 C_{*}\left\|\tilde{z}^{\alpha}(s)\right\|_{\tilde{V}} d s\right)\right) \leq \exp \left(t_{0}\left(\frac{k_{0} v}{2}-2 C_{*} E\left\|\tilde{z}^{\alpha}(0)\right\|_{\tilde{V}}\right)\right) .
$$

Moreover, thanks to (20) and (21) we have for all $\alpha \geq \alpha_{*}$

$$
E\left\|\tilde{z}^{\alpha}(0)\right\|_{\tilde{V}}<\frac{k_{0} v}{8 C_{*}} .
$$

Hence, for every $t_{0} \leq s_{0}(\omega)$ and $\alpha \geq \alpha_{*}$ one has

$$
\exp \left(t_{0}\left(\frac{k_{0} v}{2}+\frac{1}{t_{0}} \int_{t_{0}}^{0} 2 C_{*}\left\|\tilde{z}^{\alpha}(s)\right\|_{\tilde{V}} d s\right)\right) \leq \exp \left(t_{0} \frac{k_{0} v}{8 C_{*}}\right) .
$$

In addition, see [22] for more details, one can easily prove that there exists an a.s. finite random constant $C_{7}(\omega)$ such that

$$
\left|\tilde{z}^{\alpha}(t)\right|_{\tilde{H}} \leq C_{7}(\omega)|t|, \quad \text { for all } t \leq-1
$$

Let us assume that the bounded ball $B \subset \tilde{H}$ is inside a ball of radius $\rho_{1}$, then

$$
\left|\tilde{u}\left(t_{0}\right)\right|_{\tilde{H}} \leq \rho_{1}, \quad \text { for all } t_{0} \leq 0 .
$$

Hence, for every $t_{0} \leq s_{0}(\omega)$ and $\alpha \geq \alpha_{*}$

$$
\begin{aligned}
|\tilde{v}(t)|_{\tilde{H}}^{2} \leq & \left|\tilde{u}\left(t_{0}\right)\right|_{\tilde{H}}^{2} \exp \left(t_{0} \frac{k_{0} v}{8 C_{*}}\right)+\left|\tilde{z}^{\alpha}\left(t_{0}\right)\right|_{\tilde{H}}^{2} \exp \left(t_{0} \frac{k_{0} v}{8 C_{*}}\right) \\
& +\int_{t_{0}}^{t} f(s) e^{\int_{s}^{t}\left(2 C_{*}\left\|\tilde{z}^{\alpha}(r)\right\|_{\tilde{V}}-\frac{k_{0} v}{2}\right) d r} d s
\end{aligned}
$$




$$
\begin{aligned}
\leq & \left(\rho_{1}^{2}+C_{7}^{2}(\omega)\left|t_{0}\right|^{2}\right) \exp \left(t_{0} \frac{k_{0} v}{8 C_{*}}\right) \\
& +\int_{-\infty}^{t} f(s) e^{\int_{s}^{t}\left(2 C_{*}\left\|\tilde{z}^{\alpha}(r)\right\|_{\tilde{V}}-\frac{k_{0} v}{2}\right) d r} d s
\end{aligned}
$$

Now choose $s_{1}(\omega)<0$ such that $\left(\rho_{1}^{2}+C_{7}^{2}(\omega)\left|t_{0}\right|^{2}\right) \exp \left(t_{0} \frac{k_{0} v}{8 C_{*}}\right) \leq 1$, for all $t_{0} \leq s_{1}(\omega)$, and let us denote by $t_{B}(\omega)=\min \left\{s_{0}(\omega), s_{1}(\omega)\right\}$, then we get that the integral inside $R_{1}^{t}(\omega)$ is a.s. convergent and that for every $t \leq t_{B}(\omega)$

$$
|\tilde{v}(t)|_{\tilde{H}}^{2} \leq R_{1}^{t}(\omega)
$$

Hence, the ball $B\left(0, R_{1}^{t}(\omega)\right)$ is an absorbing ball at time $t$. This completes the proof.

Lemma 17 Suppose that the assumptions of Lemma 15 are satisfied, and assume that $\alpha \geq$ $\alpha_{*}$ and $\left|s_{0}(\omega)\right|$ is large enough such that (29) and (28) hold, respectively. In addition, assume that $\tilde{v}_{t_{0}} \in \tilde{V}$, then for every $t_{0} \leq t_{B}(\omega)$, there exists $R_{2}(\omega), P$ - a.s. bounded, such that

$$
\int_{-1}^{0}\|\tilde{v}(t)\|_{\tilde{V}}^{2} d t \leq R_{2}(\omega)
$$

where

$$
R_{2}(\omega):=\frac{1}{v} R_{1}^{\{t=-1\}}(\omega)+\int_{-1}^{0} f(t) d t+\int_{-1}^{0} R_{1}^{s}(\omega)\left(2 C_{*}\left\|\tilde{z}^{\alpha}(s)\right\|_{\tilde{V}}+\frac{k_{0} v}{2}\right) d s .
$$

Notice again that in principle $R_{2}(\omega)$ depends on $\alpha$, however, if we fix $\alpha=\alpha_{0}:=2 \alpha_{*}(v)$ then $R_{2}(\omega)$ will depend only on $v$ and the statement of Lemma 17 will still be valid.

Proof Integrate (27) over $(-1,0)$, then use Lemma 15 to estimate $|\tilde{v}(-1)|_{\tilde{H}}^{2}$.

Lemma 18 Assume the assumptions of Lemma 17 are satisfied, then there exists $R_{3}(\omega)$, $P$-a.s. finite such that

$$
\|\tilde{v}(0)\|_{\tilde{V}}^{2} \leq R_{3}(\omega)
$$

where

$$
\begin{aligned}
R_{3}(\omega):= & \left(R_{2}(\omega)+\int_{-1}^{0}\left(2 C_{*}^{2}\left(R_{1}^{s}(\omega)+\left|\tilde{z}^{\alpha}(s)\right|_{\tilde{H}}^{2}\right)\left\|\tilde{z}^{\alpha}(s)\right\|_{\tilde{V}}^{2}+\frac{2 \alpha^{2}}{v}\left|\tilde{z}^{\alpha}(s)\right|_{\tilde{H}}^{2}\right) d s\right) \\
& \times \exp \left(\int_{-1}^{0} 2 C_{*}^{2}\left(R_{1}^{s}(\omega)+\left|\tilde{z}^{\alpha}(s)\right|_{\tilde{H}}^{2}\right) d s\right) .
\end{aligned}
$$

Proof Let us take the inner product in $\tilde{H}$ of (22) with $\tilde{A} \tilde{v}$, we get

$$
\begin{aligned}
\frac{1}{2} \frac{d}{d t}\|\tilde{v}\|_{\tilde{V}}^{2}+v|\tilde{A} \tilde{v}|_{\tilde{H}}^{2} & =-\left\langle\tilde{B}_{\lambda}\left(\tilde{v}+\tilde{z}^{\alpha}, \tilde{v}+\tilde{z}^{\alpha}\right), \tilde{A} \tilde{v}\right\rangle+\alpha\left|\tilde{z}^{\alpha}, \tilde{A} \tilde{v}\right\rangle \\
& \leq C_{*}\left|\tilde{B}_{\lambda}\left(\tilde{v}+\tilde{z}^{\alpha}, \tilde{v}+\tilde{z}^{\alpha}\right)\right|_{\tilde{H}}|\tilde{A} \tilde{v}|_{\tilde{H}}+\alpha\left|\tilde{z}^{\alpha}\right|_{\tilde{H}}|\tilde{A} \tilde{v}|_{\tilde{H}} \\
& \leq \frac{v}{2}|\tilde{A} \tilde{v}|_{\tilde{H}}^{2}+C_{*}^{2}\left|\tilde{v}+\tilde{z}^{\alpha}\right|_{\tilde{H}}^{2}\left\|\tilde{v}+\tilde{z}^{\alpha}\right\|_{\tilde{V}}^{2}+\frac{4 \alpha^{2}}{v}\left|\tilde{z}^{\alpha}\right|_{\tilde{H}}^{2}
\end{aligned}
$$


In the above estimate, we have used Lemma 2 and the Young's inequality. Hence,

$$
\begin{aligned}
\|\tilde{v}(t)\|_{\tilde{V}}^{2} \leq & \|\tilde{v}(s)\|_{\tilde{V}}^{2}+\int_{s}^{t} 2 C_{*}^{2}\left|\tilde{v}(r)+\tilde{z}^{\alpha}(r)\right|_{\tilde{H}}^{2}\|\tilde{v}(r)\|_{\tilde{V}}^{2} d r \\
& +\int_{s}^{t}\left(2 C_{*}^{2}\left|\tilde{v}(r)+\tilde{z}^{\alpha}(r)\right|_{\tilde{H}}^{2}\left\|\tilde{z}^{\alpha}(r)\right\|_{\tilde{V}}^{2}+\frac{2 \alpha^{2}}{v}\left|\tilde{z}^{\alpha}(r)\right|_{\tilde{H}}^{2}\right) d r .
\end{aligned}
$$

Using Gronwall lemma, we get

$$
\begin{aligned}
\|\tilde{v}(t)\|_{\tilde{V}}^{2} \leq & \|\tilde{v}(s)\|_{\tilde{V}}^{2} e^{\int_{s}^{t} 2 C_{*}^{2} \tilde{v}(r)+\left.\tilde{z}^{\alpha}(r)\right|_{\tilde{H}} ^{2} d r} \\
& +\int_{s}^{t}\left(2 C_{*}^{2}\left|\tilde{v}(r)+\tilde{z}^{\alpha}(r)\right|_{\tilde{H}}^{2}\left\|\tilde{z}^{\alpha}(r)\right\|_{\tilde{V}}^{2}+\frac{2 \alpha^{2}}{v}\left|\tilde{z}^{\alpha}(r)\right|_{\tilde{H}}^{2}\right) e^{\int_{r}^{t} 2 C_{*}^{2}\left|\tilde{v}\left(r^{\prime}\right)+\tilde{z}^{\alpha}\left(r^{\prime}\right)\right|_{\tilde{H}}^{2} d r^{\prime}} d r .
\end{aligned}
$$

Therefore, for $t=0$, we have

$$
\begin{aligned}
\|\tilde{v}(0)\|_{\tilde{V}}^{2} \leq & \|\tilde{v}(s)\|_{\tilde{V}}^{2} e^{\int_{s}^{0} 2 C_{*}^{2}\left|\tilde{v}(r)+\tilde{z}^{\alpha}(r)\right|_{\tilde{H}}^{2} d r} \\
& +\int_{s}^{0}\left(2 C_{*}^{2}\left|\tilde{v}(r)+\tilde{z}^{\alpha}(r)\right|_{\tilde{H}}^{2}\left\|\tilde{z}^{\alpha}(r)\right\|_{\tilde{V}}^{2}+\frac{2 \alpha^{2}}{v}|\tilde{z}(r)|_{\tilde{H}}^{2}\right) e^{\int_{r}^{0} 2 C_{*}^{2}\left|\tilde{v}\left(r^{\prime}\right)+\tilde{z}^{\alpha}\left(r^{\prime}\right)\right|_{\tilde{H}}^{2} d r^{\prime}} d r .
\end{aligned}
$$

Now, we integrate over $(-1,0)$ to obtain

$$
\begin{aligned}
\|\tilde{v}(0)\|_{\tilde{V}}^{2} \leq & \left(\int_{-1}^{0}\|\tilde{v}(s)\|_{\tilde{V}}^{2} d s+\int_{-1}^{0}\left(2 C_{*}^{2}\left|\tilde{v}(s)+\tilde{z}(s)^{\alpha}\right|_{\tilde{H}}^{2}\left\|\tilde{z}^{\alpha}(s)\right\|_{\tilde{V}}^{2}+\frac{2 \alpha^{2}}{v}\left|\tilde{z}^{\alpha}(s)\right|_{\tilde{H}}^{2}\right) d s\right) \\
& \times \exp \left(\int_{-1}^{0} 2 C_{*}^{2}\left|\tilde{v}(s)+\tilde{z}^{\alpha}(s)\right|_{\tilde{H}}^{2} d s\right) .
\end{aligned}
$$

Consequently, we use the estimate of the preceding lemma to complete the proof.

Lemma 19 Let $\varphi^{\lambda}(t, \omega)$ be a stochastic flow associated to (9), defined on a $\theta_{t}$-invariant full measure set $\Omega_{1}^{0}$. On the $\theta_{t}$-invariant full measure set $\Omega_{W}^{0}$ described previously, one can define $\tilde{z}^{\alpha}(t, \omega)$ and $\tilde{v}\left(t, \omega, \tilde{u}_{0}\right)$, say for a given fixed $\alpha=\alpha_{0}:=2 \alpha_{*}(v)$ (see (21)). On the $\theta_{t}$-invariant full measure set $\Omega^{0}=\Omega_{1}^{0} \cap \Omega_{W}^{0}$ we have

$$
\varphi^{\lambda}(t, \omega) \tilde{u}_{0}=\tilde{v}\left(t, \omega, \tilde{u}_{0}\right)+\tilde{z}^{\alpha}(t, \omega)
$$

and for all $\omega \in \Omega^{0}$, there exists a compact absorbing set at time 0 in $\tilde{H}$ for $\varphi^{\lambda}(t, \omega)$.

Proof We have proved in Lemma 18, that the ball $B\left(0, R_{3}(\omega)\right.$ is an absorbing set at time 0 in $\tilde{V}$, which is compact in $\tilde{H}$. Hence, defining $K(\omega):=\left\{u \in \tilde{V}:\|u\|_{\tilde{V}}^{2} \leq R_{3}+\left\|\tilde{z}^{\alpha}(0, \omega)\right\|_{\tilde{V}}^{2}\right\}$ concludes the proofs.

Theorem 20 For every value of the parameter $\lambda \in \mathbb{R}$, the random dynamical system $\varphi^{\lambda}$ associated to the equation (9) has a unique global random attractor $\mathcal{A}_{\lambda}(\omega)$. 
Proof Using Theorem 11 and the existence of a compact absorbing set in $\tilde{H}$, we have the existence of a random attractor $\mathcal{A}_{\lambda}(\omega)$ which is forward invariant.

We can now apply Theorem 2 from [8]. The statement of this theorem is composed of two parts, the first one devoted to the convergence of the random attractor to the deterministic one as the intensity of the noise goes to zero; the second one to the upper semicontinuity of the random attractor when the parameter of the noise varies with continuity to some non-zero value. We apply the second part. The assumptions of the second part are: i) the existence of the random attractor for every fixed value of the parameter, ii) the $P$-a.s. continuous dependence of trajectories on the parameter, in any fixed finite interval of time, uniformly in the initial conditions taken from any fixed non-random bounded set. Both assumptions have been proved in the previous sections. Thus we get the following final result.

Theorem 21 Let $\mathcal{A}_{\lambda}(\omega)$ be the random attractor associated with equation (9), then there is upper semicontinuous convergence of $\mathcal{A}_{\lambda}(\omega)$ to $\mathcal{A}_{0}(\omega)$ as $\lambda \rightarrow 0$ :

$$
\lim _{\lambda \rightarrow 0} d_{H}\left(\mathcal{A}_{\lambda}(\omega), \mathcal{A}_{0}(\omega)\right)=0 \quad \text { with P-a.s. }
$$

\subsection{Random Squeezing Property}

In this section, we are going to establish that the random attractor of the random dynamical system $\varphi$ associated with (9) has a finite Hausdorff dimension (notice here that for simplicity of notation, we dropped the superscript $\lambda$ in $\varphi^{\lambda}$. Let $\tilde{u}$ and $\tilde{v}$ be two solutions of the associated equation (9), then the difference $\tilde{u}-\tilde{v}$ is solution of

$$
\frac{d(\tilde{u}-\tilde{v})}{d t}+v \tilde{A}(\tilde{u}-\tilde{v})=-\tilde{B}_{\lambda}(\tilde{u}, \tilde{u}-\tilde{v})-\tilde{B}_{\lambda}(\tilde{u}-\tilde{v}, \tilde{v})
$$

Lemma 22 Let $\Pi$ be the orthogonal projection on the first $n$ eigenvectors of the operator $\tilde{A}$. Then,

$$
\begin{aligned}
& \left|\Pi\left(\varphi(t, \omega) \tilde{u}_{0}-\varphi(t, \omega) \tilde{v}_{0}\right)\right|_{\tilde{H}} \leq\left|\tilde{u}_{0}-\tilde{v}_{0}\right|_{\tilde{H}} e^{\frac{C_{*}}{v} \int_{0}^{t} R_{1}\left(\theta_{s} \omega\right) d s} \\
& \left|(I-\Pi)\left(\varphi(t, \omega) \tilde{u}_{0}-\varphi(t, \omega) \tilde{v}_{0}\right)\right|_{\tilde{H}} \\
& \quad \leq\left|\tilde{u}_{0}-\tilde{v}_{0}\right|_{\tilde{H}}\left(e^{-k_{n+1} v t}+\left(\frac{\sqrt{2} C^{2}}{\left(\nu k_{n+1}\right)^{3 / 2}}\right) e^{\int_{0}^{t}\left[R_{1}\left(\theta_{s}(\omega)\right)\right]^{2}+\frac{C_{*}}{v} R_{1}\left(\theta_{s}(\omega)\right) d s}\right),
\end{aligned}
$$

where $C_{*}$ is the constant in inequality (6), and $C$ is the constant in the inequalities in Lemma 2 , for all $t \geq 0$ and all $\tilde{u}_{0}, \tilde{v}_{0} \in \mathcal{A}(\omega)$, where $R_{1}=R_{1}^{\{t=0\}}$ given in Lemma 16, say for a given fixed $\alpha=\alpha_{0}:=2 \alpha_{*}(v)($ see $(21))$.

Proof We multiply (31) by $\tilde{u}-\tilde{v}$, and use inequality (6) to obtain

$$
\begin{aligned}
\frac{1}{2} \frac{d}{d t}|(\tilde{u}-\tilde{v})|_{\tilde{H}}^{2}+v\|(\tilde{u}-\tilde{v})\|_{\tilde{V}}^{2} & \leq\left|\left\langle\left(\tilde{B}_{\lambda}(\tilde{u}-\tilde{v}, \tilde{v})\right),(\tilde{u}-\tilde{v})\right\rangle\right| \\
& \leq \frac{v}{2}\|(\tilde{u}-\tilde{v})\|_{\tilde{V}}^{2}+\frac{1}{2 v}\left\|\tilde{B}_{\lambda}(\tilde{u}-\tilde{v}, \tilde{v})\right\|_{\tilde{V}^{\prime}} \\
& \leq \frac{v}{2}\|(\tilde{u}-\tilde{v})\|_{\tilde{V}}^{2}+\frac{C_{*}}{2 v}|\tilde{u}-\tilde{v}|_{\tilde{H}}^{2}|\tilde{v}|_{\tilde{H}}^{2} .
\end{aligned}
$$


Using Gronwall lemma, we obtain

$$
|\tilde{u}(t)-\tilde{v}(t)|_{\tilde{H}}^{2} \leq\left|\tilde{u}\left(t_{0}\right)-\tilde{v}\left(t_{0}\right)\right|_{\tilde{H}}^{2} e^{\frac{C_{*}}{v} \int_{t_{0}}^{t}|\tilde{v}(s)|_{\tilde{H}}^{2} d s}
$$

Now, using the invariance of the attractor, if we take $\tilde{u}_{0}, \tilde{v}_{0} \in \mathcal{A}(\omega)$ then $\tilde{u}(t), \tilde{v}(t) \in \mathcal{A}\left(\theta_{t} \omega\right)$, and therefore by Lemmas 15 and 16 we have

$$
\left|\varphi(t, \omega) \tilde{u}_{0}-\varphi(t, \omega) \tilde{v}_{0}\right|_{\tilde{H}}^{2} \leq\left|\tilde{u}_{0}-\tilde{v}_{0}\right|_{\tilde{H}}^{2} e^{\frac{C_{*}}{v} \int_{0}^{t} R_{1}\left(\theta_{s} \omega\right) d s}
$$

for all $t \geq 0$ and all $\tilde{u}_{0}, \tilde{v}_{0} \in \mathcal{A}(\omega)$.

Recall that $\Pi$ is a projection on the n-dimensional subspace of eigenvectors of the operator $\tilde{A}$, we have

$$
\left|\Pi\left(\varphi(t, \omega) \tilde{u}_{0}-\varphi(t, \omega) \tilde{v}_{0}\right)\right|_{\tilde{H}}^{2} \leq\left|\varphi(t, \omega) \tilde{u}_{0}-\varphi(t, \omega) \tilde{v}_{0}\right|_{\tilde{H}}^{2} .
$$

Let $Q:=I-\Pi$, it commutes with $\tilde{A}$ but not with $\tilde{B}_{\lambda}$.

Let us apply the operator $Q$ to (31), then using Lemma 2 and the Poincare inequality we get

$$
\begin{aligned}
& \frac{1}{2} \frac{d}{d t}|Q(\tilde{u}-\tilde{v})|_{\tilde{H}}^{2}+v\|Q(\tilde{u}-\tilde{v})\|_{\tilde{V}}^{2} \leq\left|\left\langle Q\left(\tilde{B}_{\lambda}(\tilde{u}, \tilde{u}-\tilde{v})\right), Q(\tilde{u}-\tilde{v})\right\rangle\right| \\
& +\left|\left\langle Q\left(\tilde{B}_{\lambda}(\tilde{u}-\tilde{v}, \tilde{v})\right), Q(\tilde{u}-\tilde{v})\right\rangle\right| \\
& \leq\left(\left|\tilde{B}_{\lambda}(\tilde{u}, \tilde{u}-\tilde{v})\right|_{\tilde{H}}+\left|\tilde{B}_{\lambda}(\tilde{u}-\tilde{v}, \tilde{v})\right|_{\tilde{H}}\right)|Q(\tilde{u}-\tilde{v})|_{\tilde{H}} \\
& \leq \frac{C}{\sqrt{k_{n+1}}}|\tilde{u}-\tilde{v}|_{\tilde{H}}\left(\|\tilde{u}\|_{\tilde{V}}+\|\tilde{v}\|_{\tilde{V}}\right)\|Q(\tilde{u}-\tilde{v})\|_{\tilde{V}} \\
& \leq \frac{v}{2}\|Q(\tilde{u}-\tilde{v})\|_{\tilde{V}}^{2}+\frac{C^{2}}{2 k_{n+1} v}|\tilde{u}-\tilde{v}|_{\tilde{H}}^{2}\left(|\tilde{u}|_{\tilde{V}}^{2}+|\tilde{v}|_{\tilde{V}}^{2}\right) .
\end{aligned}
$$

Now, using the Poincare inequality on the left side of the above inequality we get that

$$
\frac{d}{d t}|Q(\tilde{u}-\tilde{v})|_{\tilde{H}}^{2}+v k_{n+1}|Q(\tilde{u}-\tilde{v})|_{\tilde{H}}^{2} \leq \frac{C^{2}}{k_{n+1} v}|\tilde{u}-\tilde{v}|_{\tilde{H}}^{2}\left(|\tilde{u}|_{\tilde{V}}^{2}+|\tilde{v}|_{\tilde{V}}^{2}\right) .
$$

Hence,

$$
\begin{aligned}
|Q(\tilde{u}-\tilde{v})(t)|_{\tilde{H}}^{2} \leq & \left|Q(\tilde{u}-\tilde{v})\left(t_{0}\right)\right|_{\tilde{H}}^{2}-k_{n+1} v \int_{t_{0}}^{t}|Q(\tilde{u}-\tilde{v})(s)|_{\tilde{H}}^{2} d s \\
& +\frac{C^{2}}{k_{n+1} v} \int_{t_{0}}^{t}|(\tilde{u}-\tilde{v})(s)|_{\tilde{H}}^{2}\left(|\tilde{u}(s)|_{\tilde{V}}^{2}+|\tilde{v}(s)|_{\tilde{V}}^{2}\right) d s
\end{aligned}
$$

Using Gronwall lemma we get

$$
\begin{aligned}
|Q(\tilde{u}-\tilde{v})(t)|_{\tilde{H}}^{2} \leq & \left|Q(\tilde{u}-\tilde{v})\left(t_{0}\right)\right|_{\tilde{H}}^{2} e^{-k_{n+1} v\left(t-t_{0}\right)} \\
& +\frac{C^{2}}{k_{n+1} v} \int_{t_{0}}^{t} e^{-k_{n+1} v(t-s)}|(\tilde{u}-\tilde{v})(s)|_{\tilde{H}}^{2}\left(|\tilde{u}(s)|_{\tilde{H}}^{2}+|\tilde{v}(s)|_{\tilde{H}}^{2}\right) d s .
\end{aligned}
$$


Let us take $t_{0}=0$ and $\tilde{u}_{0}, \tilde{v}_{0} \in \mathcal{A}(\omega)$, then we have, thanks to Lemmas 15 and 16 , that

$$
|Q(\tilde{u}-\tilde{v})(t)|_{\tilde{H}}^{2} \leq\left|\tilde{u}_{0}-\tilde{v}_{0}\right|_{\tilde{H}}^{2} e^{-k_{n+1} v t}+\frac{C^{2}}{k_{n+1} v} \int_{0}^{t} e^{-k_{n+1} v(t-s)} R_{1}\left(\theta_{s}(\omega)\right)|(\tilde{u}-\tilde{v})(s)|_{\tilde{H}}^{2} d s .
$$

We use (34) in the above inequality to obtain

$$
\begin{aligned}
& |Q(\tilde{u}-\tilde{v})(t)|_{\tilde{H}}^{2} \\
& \quad \leq\left|\tilde{u}_{0}-\tilde{v}_{0}\right|_{\tilde{H}}^{2}\left(e^{-k_{n+1} v t}+\frac{C^{2}}{k_{n+1} v} \int_{0}^{t} e^{-k_{n+1} v(t-s)} R_{1}\left(\theta_{s}(\omega)\right) e^{\int_{0}^{s} \frac{C_{*}}{v} R_{1}\left(\theta_{r}(\omega)\right) d r} d s\right) \\
& \quad \leq\left|\tilde{u}_{0}-\tilde{v}_{0}\right|_{\tilde{H}}^{2}\left(e^{-k_{n+1} v t}+\frac{C^{2}}{k_{n+1} v}\left(e^{\int_{0}^{t} \frac{C_{*}}{v} R_{1}\left(\theta_{s}(\omega)\right) d s}\right) \int_{0}^{t} e^{-k_{n+1} v(t-s)} R_{1}\left(\theta_{s}(\omega)\right) d s\right) .
\end{aligned}
$$

On the other hand using Cauchy-Schwarz inequality and the fact that $\sqrt{x} \leq e^{x}$ for all $x>0$,

$$
\begin{aligned}
\int_{0}^{t} e^{-k_{n+1} v(t-s)} R_{1}\left(\theta_{s}(\omega)\right) d s & \leq\left(\int_{0}^{t} e^{-2 k_{n+1} v(t-s)}\right)^{1 / 2}\left(\int_{0}^{t}\left[R_{1}\left(\theta_{s}(\omega)\right)\right]^{2} d s\right)^{1 / 2} \\
& \leq\left(\frac{1}{2 k_{n+1} v}\right)^{1 / 2} e^{\int_{0}^{t}\left[R_{1}\left(\theta_{s}(\omega)\right)\right]^{2} d s}
\end{aligned}
$$

Combining all the above estimates we get that

$$
|Q(\tilde{u}-\tilde{v})(t)|_{\tilde{H}}^{2} \leq\left|\tilde{u}_{0}-\tilde{v}_{0}\right|_{\tilde{H}}^{2}\left(e^{-k_{n+1} v t}+\frac{\sqrt{2} C^{2}}{\left(k_{n+1} v\right)^{3 / 2}} e^{\int_{0}^{t}\left[R_{1}\left(\theta_{s}(\omega)\right)\right]^{2}+\frac{C_{*}}{v} R_{1}\left(\theta_{s}(\omega)\right) d s}\right) .
$$

\subsection{Finite Dimensionality of the Random Attractor}

In order to be able to apply Theorem 14 we need to show that $E\left(C_{\mathcal{H}}(\omega)\right)<\infty$, where

$$
C_{\mathcal{H}}(\omega):=\left[R_{1}(\omega)\right]^{2}+\frac{C_{*}}{v} R_{1}(\omega),
$$

is the exponent in equation (33) of the squeezing Lemma 22. This is because $C_{\mathcal{H}}(\omega)$ plays, in our case, the role of $C_{5}(\omega)$ in Theorem 14. Here $R_{1}(\omega)=R_{1}^{\{t=0\}}(\omega)$ given in Lemma 16 (see also Lemma 22), say for a given fixed $\alpha=\alpha_{0}:=2 \alpha_{*}(v)$ (see (21)). In order to get the finite expectation of $C_{\mathcal{H}}$, i.e. $E\left(C_{\mathcal{H}}(\omega)\right)<\infty$, we need to estimate the moments of the radii $R_{1}$.

Proposition 23 Let $C_{*}>0$ be the constant in the inequality (6), $\gamma_{0}=v k_{0}$, and $\alpha_{0}=2 \alpha_{*}(v)$ (see (21)). Then the stationary process $\tilde{z}_{t}$ that solves of the equation

$$
d \tilde{z}_{t}=-\left(\tilde{A}+\alpha_{0}\right) \tilde{z}_{t} d t+d \tilde{W}_{t}
$$

satisfies

$$
E\left[e^{C_{*} \int_{s}^{\tau}\left\|\tilde{z}_{t}\right\|_{\tilde{V}} d t}\right] \leq \tilde{C}_{2} e^{\gamma_{0}(\tau-s)},
$$

for all $s<\tau \leq 0$, where $\tilde{C}_{2}=E\left[e^{\frac{C_{*}^{2}\left\|\tilde{z}_{0}\right\|_{\tilde{V}}^{2}}{4 \gamma_{0}}}\right]<\infty$. 
Proof By Young's inequality we have

$$
C_{*}\left\|\tilde{z}_{t}\right\|_{\tilde{V}}=2 \sqrt{\gamma_{0}(\tau-s)} \frac{C_{*}\left\|\tilde{z}_{t}\right\|_{\tilde{V}}}{2 \sqrt{\gamma_{0}(\tau-s)}} \leq \gamma_{0}(\tau-s)+\frac{C_{*}^{2}\left\|\tilde{z}_{t}\right\|_{\tilde{V}}^{2}}{4 \gamma_{0}(\tau-s)},
$$

therefore,

$$
E\left[e^{C_{*} \int_{s}^{\tau}\left\|\tilde{z}_{t}\right\|_{\tilde{V}} d t}\right] \leq e^{\gamma_{0}(\tau-s)} E\left[e^{\frac{1}{(\tau-s)} \int_{s}^{\tau} \frac{C_{*}^{2}\left\|\tilde{z}_{t}\right\|_{\tilde{V}}^{2}}{4 \gamma_{0}} d t}\right] .
$$

Thus, it is sufficient to show that

$$
E\left[e^{\frac{1}{(\tau-s)} \int_{s}^{\tau} \frac{C_{*}^{2}\left\|\tilde{z}_{z}\right\|_{\tilde{V}}^{2}}{4 \gamma_{0}} d t}\right] \leq \tilde{C}_{2} .
$$

Thanks to Jensen inequality we have

$$
E\left[e^{\frac{1}{(\tau-s)} \int_{s}^{\tau} \frac{C_{*}^{2}\left\|\tilde{z}_{t}\right\|_{\tilde{V}}^{2}}{4 \gamma_{0}} d t}\right] \leq \frac{1}{(\tau-s)} \int_{s}^{\tau} E\left[e^{\frac{C_{*}^{2}\left\|\tilde{z}_{t}\right\|_{\tilde{V}}^{2}}{4 \gamma_{0}}}\right] d t .
$$

Since $\tilde{z}_{t}$ is a stationary process then it follows that

$$
E\left[e^{\frac{C_{*}^{2}\left\|\tilde{z}_{\tilde{z}}\right\|_{\tilde{V}}^{2}}{4 \gamma_{0}}}\right]=E\left[e^{\frac{C_{*}^{2}\left\|\tilde{z}_{0}\right\|_{\tilde{V}}^{2}}{4 \gamma_{0}}}\right]<\infty,
$$

thanks to (21). The proof is complete.

Let us recall that

$$
R_{1}^{t}(\omega):=1+\int_{-\infty}^{t} f(s) e^{\int_{s}^{t}\left(2 C_{*}\|\tilde{z}(r)\|-\frac{k_{0} v}{2}\right) d r} d s
$$

where

$$
f(t):=\frac{4 C_{*}^{2}}{v}|\tilde{z}(t)|^{4}+\frac{8 \alpha^{2}}{k_{0} v}|\tilde{z}(t)|^{2},
$$

and that $R_{1}(\omega)=R_{1}^{\{t=0\}}(\omega)$, which, as it has been remarked in Sect. 4.2, depend on the parameter $\alpha$. Hereafter we choose $\alpha=\alpha_{0}=2 \alpha_{*}(v)$ (see (21) and Proposition 23). Therefore, the relevant results of Sect. 4.2 are valid for this choice of $\alpha$.

Lemma 24 Let $\gamma_{0}$ and $\alpha_{0}$ be as in Proposition 23. Then

$$
E\left(\left(R_{1}(\omega)\right)^{2}\right)<\infty
$$

and by the Cauchy-Schwarz inequality

$$
E R_{1}(\omega)<\infty .
$$

Consequently,

$$
E\left(C_{\mathcal{H}}\right)<\infty,
$$

where $C_{\mathcal{H}}(\omega)$ is given in (35). 
Proof First we observe that using successively Jensen inequality, Fubini Theorem and Hölder inequality yield

$$
\begin{aligned}
E\left(R_{1}(\omega)\right)^{2} & =E\left(1+\int_{-\infty}^{0} f(s) e^{\int_{s}^{0}\left(2 C_{*}\|\tilde{z}(r)\|-\frac{k_{0} v}{2}\right) d r} d s\right)^{2} \\
& \leq 2+E\left(\int_{-\infty}^{0} f(s) e^{\int_{s}^{0}\left(2 C_{*}\|\tilde{z}(r)\|-\frac{k_{0} v}{2}\right) d r} d s\right)^{2} \\
& \leq 2+E \int_{-\infty}^{0} f(s)^{2} e^{2 \int_{s}^{0}\left(2 C_{*}\|z(r)\|-\frac{k_{0} v}{2}\right) d r} d s \\
& \leq 2+\int_{-\infty}^{0} E\left(f(s)^{2} e^{2 \int_{s}^{0}\left(2 C_{*}\|\tilde{z}(r)\|-\frac{k_{0} v}{2}\right) d r}\right) d s \\
& \leq 2+\int_{-\infty}^{0}\left(E f(s)^{4}\right)^{1 / 2}\left(E e^{4 \int_{s}^{0}\left(2 C_{*}\|\tilde{z}(r)\|-\frac{k_{0} v}{2}\right) d r}\right)^{1 / 2} d s
\end{aligned}
$$

The process $\tilde{z}$ is stationary, thus

$$
E\left(f(s)^{4}\right)=E\left(f(0)^{4}\right) .
$$

All moment of a Gaussian random variable are finite, hence

$$
E\left(f(0)^{4}\right)<\infty
$$

Now, using the preceding estimates and Proposition 23 we conclude that

$$
\begin{aligned}
E\left(R_{1}(\omega)\right)^{2} & \leq 2+\left(E f(0)^{4}\right)^{1 / 2} \int_{-\infty}^{0}\left(E e^{\int_{s}^{0}\left(8 C_{*}\|\tilde{z}(r)\|-2 k_{0} v\right) d r}\right)^{1 / 2} d s \\
& \leq 2+\left(E f(0)^{4}\right)^{1 / 2} \int_{-\infty}^{0} e^{k_{0} v s}\left(E e^{\int_{s}^{0} 8 C_{*}\|\tilde{z}(r)\| d r}\right)^{1 / 2} d s \\
& \leq 2+\sqrt{\tilde{C}_{2}}\left(E f(0)^{4}\right)^{1 / 2} \int_{-\infty}^{0} e^{\left(k_{0} v-\frac{\gamma_{0}}{2}\right) s} d s
\end{aligned}
$$

Since $\gamma_{0}=v k_{0}$, then

$$
E\left(\left(R_{1}(\omega)\right)^{2}\right)<\infty
$$

and the proof is complete.

As a consequence, we have the following theorem

Theorem 25 Let $K_{1}, K_{2}$ and $K_{3}$ be the absolute constant stated in Theorem 14. Let $n$ be large enough such that

$$
\frac{\sqrt{2} C^{2}}{\left(v k_{n+1}\right)^{3 / 2}} \leq K_{1}, \quad \text { and } \quad k_{n+1} v \geq K_{2} E\left(C_{\mathcal{H}}\right)
$$


where $C_{\mathcal{H}}$ is given in (35), for $\alpha=\alpha_{0}=\alpha_{*}(v)$ (see (21)). Then, $P$-a.s. the random attractor $\mathcal{A}_{\lambda}(\omega)$ of the random dynamical system $\varphi^{\lambda}$ associated with (9) has finite Hausdorff dimension which is less than $K_{3} n \ln n$.

Proof The proof follows from applying Theorem 14 for $\mu=\frac{\sqrt{2} C^{2}}{\left(v k_{n+1}\right)^{3 / 2}}$ and $\delta=k_{n+1} v$. Then by virtue of (36) all the assumptions of Theorem 14 are satisfied. Hence, we get that $P$-a.s. the random attractor $\mathcal{A}_{\lambda}(\omega)$ of the random dynamical system $\varphi^{\lambda}$ associated with (9) has finite Hausdorff dimension which is less than $K_{3} n \ln n$. This completes the proof.

Acknowledgements The work of H. Bessaih was supported in part by the National Science Foundation (NSF) grand No. DMS-0608494. The work of E.S. Titi was supported in part by the NSF grant No. DMS0708832, and the ISF grant No. 120/6.

Open Access This article is distributed under the terms of the Creative Commons Attribution Noncommercial License which permits any noncommercial use, distribution, and reproduction in any medium, provided the original author(s) and source are credited.

\section{References}

1. Angheluta, L., Benzi, R., Biferale, L., Procaccia, I., Toschi, T.: Anomalous scaling exponents in nonlinear models of turbulence. Phys. Rev. Lett. 97(16), 160601 (2006)

2. Arad, I., Biferale, L., Celani, A., Procaccia, I., Vergassola, M.: Statistical conservation laws in turbulent transport. Phys. Rev. Lett. 87, 164502 (2001)

3. Arnold, L.: Random Dynamical Systems. Springer Monographs in Mathematics. Springer, Berlin (1998)

4. Barbato, D., Barsanti, M., Bessaih, H., Flandoli, F.: Some rigorous results for a stochastic Goy model. J. Stat. Phys. 125(3), 677-716 (2006)

5. Benzi, R., Levant, B., Procaccia, I., Titi, E.S.: Statistical properties of nonlinear shell models of turbulence from linear advection model: rigorous results. Nonlinearity 20(6), 1431-1441 (2007)

6. Berselli, L.C., Flandoli, F.: Remarks on determining projections for stochastic dissipative equations. Discrete Contin. Dyn. Syst. 5(1), 197-214 (1999)

7. Biferale, L.: Shell models of energy cascade in turbulence. Annu. Rev. Fluid Mech. 35, 441-468 (2003)

8. Caraballo, T., Langa, J.A., Robinson, J.C.: Upper semicontinuity of attractors for small random perturbations of dynamical systems. Commun. Part. Differ. Equ. 23(9), 1557-1581 (1998)

9. Cheban, D.N., Kloeden, P.E., Schmalfuß, B.: The relationship between pullback, forward and global attractors of nonautonomous dynamical systems. Nonlinear Dyn. Syst. Theory 2(2), 125-144 (2002)

10. Cohen, Y., Gilbert, T., Procaccia, I.: Statistically preserved structures in shell models of passive scalar advection. Phys. Rev. E. 65, 026314 (2002)

11. Constantin, P., Foias, C.: Navier-Stokes Equations. The University of Chicago Press, Chicago (1988)

12. Constantin, P., Levant, B., Titi, E.S.: Analytic study of shell models of turbulence. Physica D 219(2), 120-141 (2006)

13. Constantin, P., Levant, B., Titi, E.S.: Sharp lower bounds for the dimension of the global attractor of the Sabra shell model of turbulence. J. Stat. Phys. 127(6), 1173-1192 (2007)

14. Constantin, P., Levant, B., Titi, E.: Regularity of inviscid shell models of turbulence. Phys. Rev. E (3) 75(1), 016304 (2007)

15. Constantin, P., Foias, C., Nicolaenko, B., Temam, R.: Integral Manifolds and Inertial Manifolds for Dissipative Partial Differential Equations. Applied Mathematics Sciences, vol. 70. Springer, Berlin (1988)

16. Crauel, H.: Global random attractors are uniquely determined by attracting deterministic compact sets. Ann. Mat. Pura Appl. (4) 176, 57-72 (1999)

17. Crauel, H., Debussche, A., Flandoli, F.: Random attractors. J. Dyn. Differ. Equ. 9(2), 307-341 (1997)

18. Crauel, H., Flandoli, F.: Attractors for random dynamical systems. Probab. Theory Relat. Fields 100, 365-393 (1994)

19. Da Prato, G., Zabczyk, J.: Stochastic Equations in Infinite Dimensions. Cambridge University Press, Cambridge (1992)

20. Debussche, A.: On the finite dimensionality of random attractors. Stoch. Anal. Appl. 15(4), 473-491 (1997)

21. Debussche, A.: Hausdorff dimension of a random invariant set. J. Math. Pures Appl. (9) 77(10), 967-988 (1998) 
22. Flandoli, F.: Dissipativity and invariant measures for stochastic Navier-Stokes equations. NoDEA Nonlinear Differ. Equ. Appl. 1(4), 403-423 (1994)

23. Flandoli, F.: Stochastic differential equations in fluid dynamics. Rend. Semin. Mat. Fis. Milano 66, 121148 (1996) (1998)

24. Flandoli, F.: An Introduction to 3D Stochastic Fluid Dynamics, CIME Lecture Notes (2005)

25. Flandoli, F., Langa, J.A.: On determining modes for dissipative random dynamical systems. Stoch. Stoch. Rep. 66, 1-25 (1999)

26. Foias, C., Temam, R.: Some analytic and geometric properties of the solutions of the evolution NavierStokes equations. J. Math. Pure Appl. 58, 339-368 (1979)

27. Frisch, U.: Turbulence. Cambridge University Press, Cambridge (1995)

28. Gallavotti, G.: Foundations of Fluid Dynamics. Texts and Monographs in Physics. Springer, Berlin (2002). Translated from the Italian

29. Gledzer, E.B.: System of hydrodynamic type admitting two quadratic integrals of motion. Sov. Phys. Dokl. 18, 216-217 (1973)

30. Hale, J.: Asymptotic Behavior of Dissipative Systems. Mathematical Surveys and Monographs, vol. 25. Am. Math. Soc., Providence (1988)

31. Ladyzhenskaya, O.A.: Attractors for Semigroups and Evolution Equations. Cambridge University Press, Cambridge (1991)

32. L'vov, V.S., Podivilov, E., Pomyalov, A., Procaccia, I., Vandembroucq, D.: Improved shell model of turbulence. Phys. Rev. E 58(2), 1811-1822 (1998)

33. Pazy, A.: Semigroups of Linear Operators and Applications to Partial Differential Equations. Applied Mathematical Sciences, vol. 44. Springer, New York (1983)

34. Temam, R.: Navier-Stokes Equations. Theory and Numerical Analysis. Studies in Mathematics and its Applications, vol. 2. North-Holland, Amsterdam (1979)

35. Yamada, M., Ohkitani, K.: Temporal intermittency in the energy cascade process and local Lyapunov analysis in fully developed model of turbulence. Prog. Theor. Phys. 89, 329-341 (1989) 